\title{
Palaemonoid shrimps from the Dampier Archipelago (Crustacea: Decapoda), with a review of the Western Australian pontoniine shrimp fauna
}

\author{
A.J. Bruce \\ Queensland Museum, P.O. Box 3300, South Brisbane, Queensland, 4101 Australia. \\ email: abrucectobroad netau
}

\begin{abstract}
Palaemonoid shrimps collected in the course of the Western Australian Museum 1998-2002 Dampier Archipelago Survey are reported upon. Thirty one taxa were found, all except three belonging to the subfamily Pontoniinae. One species of pontonime shrimp is new: Periclimenes burrup sp. nov is described and illustrated, and seven are new to the Western Australian fauna. The pontoniine shrimp fauna of Western Australia is summarised.
\end{abstract}

\section{INTRODUCTION}

The marine palaemonoid fauna of Western Australia has attracted less attention than that of Australia's eastern seabord. The fauna is dominated by shrimps of the subfamily Pontoniinae, usually small, often cryptic, and frequently inconspicuous associates of other marine invertebrate hosts, particularly sponges, coelenterates, echinoderms and sea squirts.

The first pontoniine shrimp recorded from Western Australia was Anchistus custos reported by Miers (1884) (as Harpilius inermis), from Shark Bay, collected during the voyage of H.M.S Alert, 1881-2. Mary Rathbun, in 1914, again recorded Anchistus custos (as Anchistus inermis) and Periclimenes brevicarpalis (as $P$. hermitensis) from the Monte Bello Islands. Later, Balss (1921), reporting on the results of Dr E. Mjöberg's Swedish Scientific Expedition to Australia, 1910-1914, recorded Palaemonella tenuipes, Periclimenaeus hecate (as ?Coralliocaris hecate) and Periclimenes incertus (as Palaemonella biunguiculata). Further studies had to wait until the second half of the $20^{\text {th }}$ century. Recently Berggren $(1997 \mathrm{a}, \mathrm{b}, \mathrm{c})$ has studied the Western Australian caridean fauna and collected much new date, but unfortunately these collections remain unpublished. He has kindly provided access to this data, some of which is included in this report.

Prior to the present study, 52 pontoniine species were formally known from Western Australia, with a further 19 in unpublished reports. The present report adds a further 10 species to the Western Australian pontoniine fauna, all previously known from elsewhere in Australia, and one new species, making a total of 82 species ( 85 taxa). A checklist of the pontoniine shrimps now known from Western Australia is provided and the results are summarised in Table 1.

Many more pontoniine (and other) shrimp species may be expected to be found in the future in Western Australian waters. The present total may be compared with that of a single small reef on the southern Great Barrier Reef, Heron I., with over 100 species (Bruce, 1981).

\section{MATERIALS AND METHODS}

Restricted synonymies only are provided, primarily indicating name changes and major redescriptions of early described species. Full synonymies are to be found in $\mathrm{Li}(2000)$ and Davie (2002). CL refers to the postorbital carapace length; C. for Cape; coll. to collected by; I. to Island, Is for Islands; ovig. for ovigerous; Pen. for Peninsula; $\mathrm{Pt}$ for Point; spm for specimen; stn for station.

The specimens are held in the collections of the Queensland Museum (QM), the Rijksmuseum von Natuurlijke Historie, Leiden, The Netherlands (RMNH) and the Western Australian Museum, Perth (WAM).

\section{LIST OF TAXA EXAMINED}

* New to the Western Australian fauna

1. Anchistioides compressus Paul'son, 1875*

2. Anchistioides willeyi (Borradaile, 1900)*

3. Palaemonetes atrinubes Bray, 1976

4. Conchodytes meleagrinae Peters, 1852

5. Conchodytes monodactylus Holthuis, 1952

6. Coralliocaris nudirostris (Heller, 1861)*

7. Coralliocaris viridis Bruce, 1974 
8. Harpiliopsis beaupresii (Audouin, 1825)

9. Harpilius bayeri (Holthuis, 1981)

10. Kemponia amymone (De Man, 1902)

11. Kemponia andamanensis (Kemp, 1922)*

12. Kemponia elegans (Paul'son, 1875)

13. Kemponia grandis (Stimpson, 1860)

14. Kemponia aff. grandis (Stimpson, 1860)

15. Palaemonella pottsi (Borradaile, 1915)

16. Palaemonella rotumana (Borradaile, 1898)

17. Palaemonella spinulata Yokoya, $1936^{*}$

18. Palaemonella sp.

19. Periclimenaeus arabicus (Calman, 1939)

20. Periclimenaeus hecate (Nobili, 1904)

21. Periclimenella spinifera (De Man, 1902)

22. Periclimenes affinis (Zehntner, 1894)*

23. Periclimenes alegrias Bruce, 1986

24. Periclimenes burrup sp. nov.

25. Periclimenes holthuisi Bruce, 1969*

26. Periclimenes incertus Borradaile, 1915

27. Periclimenes magnificus Bruce, 1979*

28. Periclimenes novaffinis Bruce and Coombes, $1997^{*}$

29. Periclimenes soror Nobili, 1904

30. Philarius gerlachei (Nobili, 1905)

31. Philarius imperialis (Kubo, 1940)*

\section{SYSTEMATICS}

Order DECAPODA Latreille, 1802

Infraorder Caridea Dana, 1852

Superfamily Palaemonoidea Rafinesque, 1815

Family Anchistioididae Borradaile, 1915

Genus Anchistioides Paul'son, 1875

Anchistioides compressus Paul'son, 1875

Anchistioides compressus Paul'son, 1875: 115, pl. 19, fig. 5. - Davie, 2002: 220.

Material examined

Western Australia, Dampier Archipelago. WAM C 25839 (1 ovig. female), stn DA1/98/01, Dolphin I. $\left(20^{\circ} 25.852^{\prime} \mathrm{S}, 116^{\circ} 52.953^{\prime} \mathrm{E}\right), 3.0-6.5 \mathrm{~m}, 17.10 .1998$.

\section{Host}

Unidentified purple sponge.

\section{Remarks}

This is a new record for Western Australia. The specimens present no special features. Rostral dentition $11 / 9$.

\section{Australian Distribution}

Queensland. Heron I., Capricorn Is (Bruce, 1981); Heron I., Wilson I. and Moreton Bay (Bruce, 1983).

\section{Further Distribution}

Type locality: Red Sea. Also known from Zanzibar, Kenya, Andaman Islands, Japan, South China Sea, Australia and Tuamotu Islands.

\section{Anchistioides willeyi (Borradaile, 1900)}

Palaemonopsis willeyi Borradaile, 1900: 410, pls 36, 37 , fig. 7 .

Amphipalaemon willeyi - Borradaile, 1917: 407, pl. 59, fig. 13.

Anchistioides willeyi. - Gordon, 1935: 435, figs 23a, 24a. - Holthuis, 1952: 18, 214, figs 106, 107. - Bruce, 1991: 269, figs 3g, 29, 30. - Davie, 2002: 221.

\section{Material examined}

Western Australia, Dampier Archipelago. WAM C 29238 (1 spm), stn DA2/99/68, off Bluff Pt, Enderby I. $\left(20^{\circ} 40.93^{\prime} \mathrm{S}, 116^{\circ} 33.21^{\prime} \mathrm{E}\right.$ to $20^{\circ} 40.63^{\prime} \mathrm{S}$, $\left.116^{\circ} 33.36^{\prime} \mathrm{E}\right)$, rake box dredge, 9.0-9.2 m, 23.07.1999.

\section{Remarks}

Previously reported specimens exhibit two morphological types of second pereiopod chelae, long fingered and short fingered (Bruce, 1991). The present material belongs to the long fingered form, with a rostral dentition of $9 / 4$. The species is a sponge associate and has been reported from depths to $127 \mathrm{~m}$ (Bruce, 1991).

This is a new record for Western Australia.

\section{Australian Distribution}

Northern Territory: Darwin Harbour (Bruce, 1988a). Queensland: Capricorn Is; Moreton Bay (Bruce, 1983a).

\section{Further Distribution}

Type locality: Ralun, New Britain. Known also from Kenya, Zanzibar, Tanganyika, Madagascar, Maldive Islands, Singapore, Borneo, Indonesia, South China Sea, Philippines, New Caledonia and Chesterfield Islands.

\section{Family Palaemonidae Rafinesque, 1815}

Subfamily Palaemoninae Rafinesque, 1815

Genus Palaemonetes Heller, 1869

Palaemonetes atrinubes Bray, 1976

Palaemonetes atrinubes Bray, 1976: 65, figs 23-42. Davie, 2002: 301.

\section{Material examined}

Western Australia, Dampier Archipelago. WAM C 29216 (1 ovig. female), stn DA3/99/62, East Lewis I. $\left(20^{\circ} 37.499^{\prime} \mathrm{S}, 116^{\circ} 39.182^{\prime} \mathrm{E}\right)$, intertidal, 05.09.1999. 


\section{Remarks}

The specimen, with a rostral dentition of $1+6 / 4$, presents no special features.

\section{Australian Distribution}

Western Australia. Swan River; Exmouth Gulf; to Leschenault Inlet (Bray, 1976); West Governor I., Napier Broome Bay, eastern Kimberleys (Davie and Short, 1996).

\section{Further Distribution}

Type locality: Canning Bridge, Lower Swan River, Cockatoo Island. Also known from New Caledonia.

\section{Subfamily Pontoniinae Kingsley, 1878}

\section{Genus Conchodytes Peters, 1852}

\section{Conchodytes meleagrinae Peters, 1852}

Conchodytes meleagrinae Peters, 1852: 594. - Li, 2000: 25, fig. 26. - Davie, 2002: 307.

\section{Material examined}

Western Australia, Dampier Archipelago. WAM C 25302 (1 male, 1 ovig. female), stn DA1/98/03, Legendre I. $\left(20^{\circ} 24.320^{\prime} \mathrm{S}, 116^{\circ} 56.108^{\prime} \mathrm{E}\right), 2.0-15.0 \mathrm{~m}$, 18.10 .1998

\section{Host}

Pinctada margaritifera (Linnaeus, 1758), Black lipped pearl oyster [Bivalvia, Pteriidae].

\section{Remarks}

It seems remarkable that there appear to be no previous records of this well known species from Western Australia in view of the long history of the pearling industry in that state. Jones and Morgan (2002) provide a photograph of Conchodytes sp., but without indication of the locality of capture. The specimen, which may be of C. melaegrinae, has not been available for examination

This is the first published recored of this species for Western Australia.

\section{Australian Distribution}

Northern Territory: Bathhurst I. (Shiino, 1942). Queensland: Torres Straits, Warrior Reef (Miers, 1884); Torres Straits (Bate, 1888); Thursday I. (Saville Kent, 1893, as Alpheus avarus); North West I., Capricorn Is, (McNeill, 1926, as Pontonia tridacnae); Swain Reefs (McMichael, 1963); Gillet Cay; One Tree I., Swain Reefs, (Bruce, 1977); Lodestone Reef, 5 m; Tijou Reef, 2 m (Zann, 1980); Heron I., Capricorn Is (Bruce, 1981).

\section{Further Distribution}

Type locality: Ibo, Moçambique. Reported from Egypt, Yemen, Kenya, Moçambique, Madagascar, Seychelle Islands, Oman, Maldive Islands, Sri Lanka, Andaman Islands, Malaysia, Indonesia, Vietnam, China, Japan, Papua New Guinea, Caroline Islands, Marshall Islands, New Caledonia, Fijian Islands, Cook Islands, Tuamotu Islands, and Hawaiian Islands.

\section{Conchodytes monodactylus Holthuis, 1952}

Conchodytes monodactylus Holthuis, 1952: 200, figs 96-98. - Li, 2000: 26, fig. 27. - Davie, 2002: 308.

\section{Material examined}

Western Australia, Dampier Archipelago. WAM C 28058 (1male, 1 ovig. female), stn DA3/99/39, Brigadier I. $\left(20^{\circ} 25.411^{\prime} \mathrm{S}, 116^{\circ} 37.578^{\prime} \mathrm{E}\right), 15.0-27.0 \mathrm{~m}$, 28.08.1999.

\section{Host}

Pinna deltodes Menke, 1843 [Bivalvia, Pinnidae].

\section{Remarks}

Third pereiopod dactyls with rounded basal process, in male and female, as in material from Papua New Guinea (De Grave, 1998) but without small acute denticle present in types.

This is a new record for Western Australia.

\section{Australian Distribution}

Western Australia. Reported from Shark Bay by Bergren (1997b). Queensland: Magnetic I., Horseshoe Bay (Bruce, 1977c). Northern Territory: Cobourg Pen., Sandy I. No. 2 (Bruce, 1983d); Cobourg Pen. (Bruce and Coombes, 1995).

\section{Further Distribution}

Type locality: Kao Hsiung, Taiwan. Reported from Singapore, Indonesia, Hong Kong, Taiwan and Papua New Guinea.

\section{Genus Coralliocaris Stimpson, 1869}

Coralliocaris nudirostris (Heller, 1861)

Oedipus nudirostris Heller, 1861: 27; 1862: 279, pl. 3 fig. 25.

Coralliocaris nudirostris. - Borradaile, 1898: 385

Coralliocaris tahitoej Boone, 1935: 180, pl. 49 fig. 12.

Coralliocaris nudirostris. - Li, 2000: 34, fig. 34.

Coralliocaris nudirostris. - Marin et al., 2005: 201, fig. $2 a-n$. 
Table 1

\section{PONTONIINE SHRIMP SPECIES \\ REPORTED FROM WESTERN AUSTRALIA}

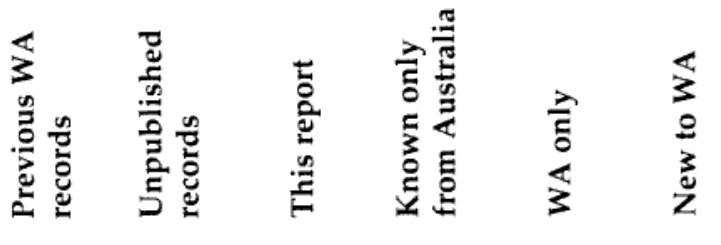

1. Anchistus custos (Forsskăl, 1775)

2. Anchistus miersi (De Man, 1888)

3. Apopontonia orbitospinata (Bruce, 1988)

4. Araiopontonia odontorhyncha Fujino and Miyake, 1970

5. Carinopontonia paucipes Bruce, 1988

6. Conchodytes biunguiculatus (Paulson, 1875)

7. Conchodytes meleagrinae Peters, 1852

8. Conchodytes maculatus Bruce, 1989

9. Conchodytes monodactylus Holthuis, 1952

10. Conchodytes tridacnae Peters, 1852

11. Coralliocaris graminea (Dana, 1852)

12. Coralliocaris nudirostris (Heller, 1861)

13. Coralliocaris viridis Bruce, 1974

14. Dasella ansoni Bruce, 1983

15. Dasycaris zanzibarica Bruce, 1969

16. Exoclimenella maldivensis Duris and Bruce, 1995

17. Exopontonia malleatrix Bruce, 1988

18. Hamodactylus aqabai Bruce and Svoboda, 1983

19. Hamodactylus boschmai Holthuis, 1952

20. Hamodactylus noumeae Bruce, 1970

21. Hamopontonia corallicola Bruce, 1970

22. Hamopontonia aff. corallicola (see Berggren, 1997c)

23. Harpiliopsis beaupresii (Audouin, 1825)

24. Harpilius bayeri (Holthuis, 1981)

25. Harpilius consobrinus (De Man, 1902)

26. Ischnopontonia lophos (Barnard, 1962)

27. Kemponia amymone (De Man, 1902)

28. Kemponia anacanthus (Bruce, 1989)

29. Kemponia andamanensis (Kemp, 1922)

30. Kemponia elegans (Paulson, 1875)

31. Kemponia grandis (Stimpson, 1860)

32. Kemponia sp., aff. grandis

33. Kemponia sp. aff. suvadivensis (see Davie and Short, 1995)

34. Kemponia tenuipes (Borradaile, 1898)

35. Manipontonia psamathe (De Man, 1902)

36. Notopontonia platycheles Bruce, 1991

37. Palaemonella crosnieri Bruce, 1978

38. Palaemonella foresti Bruce, 2002

39. Palaemonella pottsi Borradaile, 1915

40. Palaemonella rotumana (Borradaile, 1898)

41. Palaemonella spinulata Yokoya, 1936

42. Palaemonella tenuipes Dana, 1852

43. Parapontonia nudirostris Bruce, 1968

44. Periclimenaeus arabicus (Calman, 1939)

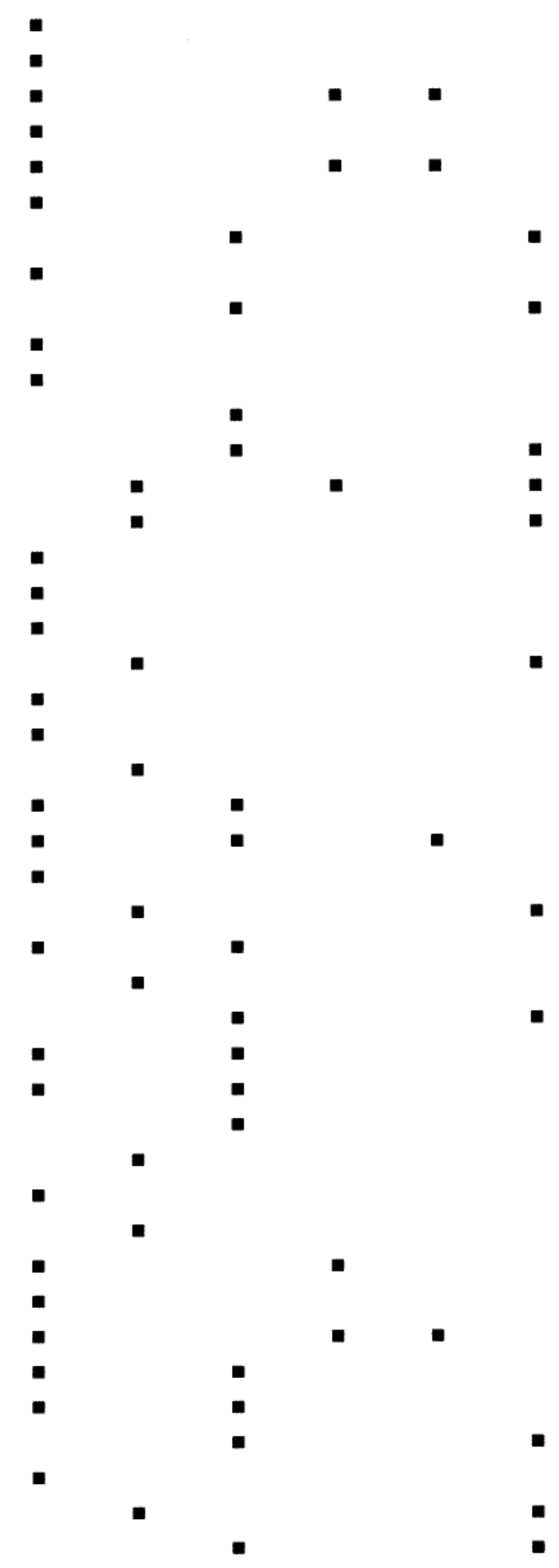


Table 1 (cont.)

\section{PONTONIINE SHRIMP SPECIES REPORTED FROM WESTERN} AUSTRALIA

45. Periclimenaeus bidentatus Bruce, 1970

46. Periclimenaeus hecate (Nobili, 1904)

47. Periclimenaeus minutus Holthuis, 1952

48. Periclimenaeus pachydentatus Bruce, 1969

49. Periclimenella spinitera (De Man, 1902)

50. Periclimenes aesopius (Bate, 1863)

51. Periclimenes affinis (Zehntner, 1894)

52. Periclimenes alegrias Bruce, 1986

53. Periclimenes amboinensis (De Man, 1888)

54. Periclimenes batei Holthuis, 1959

55. Periclimenes brevicarpalis (Schenkel, 1902)

56. Periclimenes burrup sp. nov.

57. Periclimenes commensalis Borradaile, 1915

58. Periclimenes aff. cristimanus (see Berggren, 1997c)

59. Periclimenes holthuisi Bruce, 1969

60. Periclimenes hongkongensis Bruce, 1969

61. Periclimenes imperator Bruce, 1967

62. Periclimenes indicus (Kemp, 1915)

63. Periclimenes incertus Borradaile, 1915

64. Periclimenes inornatus Kemp, 1922

65. Periclimenes kempi Bruce, 1969

66. Periclimenaeus kottae Bruce, 2005

67. Periclimenes magnificus Bruce, 1979

68. Periclimenes mahei Bruce, 1969

69. Periclimenaeus matherae Bruce, 2005

70. Periclimenes novaffinis Bruce and Coombes, 1997

71. Periclimenes aff. obscurus (see Berggren, 1997c)

72. Periclimenes seychellensis Borradaile, 1915

73. Periclimenes soror Nobili, 1904

74. Periclimenes venustus Bruce, 1990

75. Periclimenes zanzibaricus Bruce, 1967

76. Periclimenoides odontodactylus Fujino and Mivake, 1968

77. Philarius gerlachei (Nobili, 1905)

78. Philarius imperialis (Kubo, 1940)

79. Platycaris latirostris Holthuis, 1952

80. Platypontonia hvotis Hipeau-Jacquotte, 1971

81. Pontoniopsis comanthi Borradaile, 1915

82. Thaumastocaris streptopus Kemp, 1922

83. Typton nanus Bruce, 1987

84. Typtonychus dimorphus (Bruce, 1986)*

85. Vir philippinensis Bruce and Svoboda, 1984

* This taxon is now considered to be polyspecific 


\section{Material examined}

Western Australia, Dampier Archipelago. WAM C 25814 (1 ovig. female), stn DA1/98/03, Legendre I., $\left(20^{\circ} 24.320^{\prime} \mathrm{S}, 116^{\circ} 56.108^{\prime} \mathrm{E}\right), 2.0-15.0 \mathrm{~m}$, 18.08.1998.

\section{Remarks}

The single specimen has the body very broad and depressed. The rostrum is slender, slightly upcurved distally, and exceeds the proximal segment of the antennular peduncle, reaching to ca 0.75 of the intermediate segment length, distally acute, dentition $0 / 0$, without setation. The first pereiopod has the fingers of the chela ca 0.4 of the palm length, and medially concave. The specimen unfortunately lacks both second pereiopods, so the identification must be considered provisional. There is no trace of any colour pattern.

This is a new record for Western Australia.

\section{Australian Distribution}

Queensland: Orpheus and Lodestone (?) Is, Palm Is (A. hyacinthus, millepora?) (Vytopil and Willis, 2001).

\section{Further Distribution}

Type locality: Red Sea. Reported from Red Sea, Kenya, Zanzibar, Tanganyika, La Réunion, Mauritius, Seychelle Islands, Maldive Islands, Vietnam, Japan, Marshall Islands, Kiribati, and Society Islands.

\section{Coralliocaris viridis Bruce, 1974}

Coralliocaris viridis Bruce, 1974: 222, fig. 1. - Li, 2000: 38, fig. 38. - Davie, 2002: 309.

\section{Material examined}

Western Australia, Dampier Archipelago. (1) WAM C 28050 (1 spm), stn DA3/99/36, Malus I. (20³0.050'S, $\left.116^{\circ} 40.594^{\prime} \mathrm{E}\right), 6.0-14.0 \mathrm{~m}, 27.08 .1999$;

(2) WAM C 29214 (1 ovig. female), stn DA3/99/42, Georgeff Reef ( $\left.20^{\circ} 29.339^{\prime} S, 116^{\circ} 36.798^{\prime} \mathrm{E}\right)$, intertidal, 28.08.1999.

\section{Host}

Acropora sp. (1) [Scleractinia, Acroporidae].

\section{Remarks}

Specimen (1) is preserved with a pair of detached second pereiopods that are certainly not those of a Coralliocaris species. These appear identical with the chelae of Philarius gerlachei (Nobili) as illustrated by Kemp (1922, figure 75, as Harpilius gerlachei), another common associate of Acropora coral hosts (see below). The specimen has a well developed rostrum, extending well beyond the antennular peduncle, with 5 small acute similar dorsal teeth and 1 smaller ventral tooth, more closely resembling the rostrum of $C$. viridis Bruce than any other species.

Specimen (2) has a slender rostrum, reaching to distal margin of intermediate segment of the antennular peduncle, ca 0.75 of $\mathrm{CL}$, with a rostral dentition of $5 / 2$, dorsal teeth of medium size. It is somewhat intermediate between typical $C$. viridis and $C$. graminea. The colour pattern is unknown. The single small second pereiopod is ca 1.2 times the CL and is probably in the process of regeneration after autotomy.

This is a new record for Western Australia.

\section{Australian Distribution}

Western Australia. Reported from Shark Bay by Bergren (1997b). Northern Territory: Coral Bay; Orontes Reef; Oxley I., Cobourg Pen. (Bruce and Coombes, 1995). Queensland: One Tree I., Capricorn Is (Bruce, 1977a, 1983); Heron I., Capricorn Is (Bruce, 1981; 1983).

\section{Further Distribution}

Type locality: Mombasa Island, Kenya. Also reported from Moçambique, Seychelle Islands, Maldive Islands, Sri Lanka, Indonesia, Vietnam, Ryukyu Islands, Japan, Papua New Guinea and Tuamotu Islands.

\section{Genus Harpiliopsis Borradaile, 1917}

\section{Harpiliopsis beaupresii (Audouin, 1826)} Figure 1A

Palaemon Beaupresii Audouin, 1826: 91.

Harpilius Beaupresii. - Heller, 1861: 27. Borradaile, 1917: 324, 379, pl. 55 fig. 21. - Davie, 2002: 312 .

\section{Material examined}

Western Australia, Dampier Archipelago: (1) WAM C 25879 (1 male), stn DA1/98/06, Haüy I. (20²5.725'S, 116 $\left.57.580^{\circ} \mathrm{E}\right), 0.5-2.0 \mathrm{~m}, 19.10 .1998 ;(2)$ WAM C 25855 (1 ovig. female), stn DA1/98/06, Haüy I. (20²5.725'S, $\left.116^{\circ} 57.580^{\prime} \mathrm{E}\right), 0.5-2.0 \mathrm{~m}$, 19.10.1998; (3) WAM C 28078 (1 ovig. female), stn DA3/99/14, Unnamed I. (20²6.581'S, $\left.116^{\circ} 48.790^{\prime} \mathrm{E}\right)$, intertidal, 22.10.1998; (4) WAM C 29213 (1male, 1 ovig. female), stn DA3/99/36, Malus I. (20³0.050'S, $\left.116^{\circ} 40.594^{\prime} \mathrm{E}\right), 6.0-14.0 \mathrm{~m}, 27.08 .1999$.

\section{Host}

Pocillopora sp., including $P$. damicornis (4) [Scleractinia, Pocilloporidae].

\section{Remarks}

The specimen (1) has the appendix masculina (Figure 1A) on the second pleopod short, not 
exceeding the appendix interna, slightly curved medially, with ca 12 pairs of short slender simple spines along the ventrolateral margin, generally similar to that of $H$. depressus. The specimen (1), lacking both second pereiopods, was found in association with Periclimenes amymone.

\section{Australian Distribution}

Western Australia: Pt Quobba and Rottnest I. (Prince and Black, 1983); Quobba (Jones, 1990); Cartier Reef (Bruce, 1992); Rottnest I. (Jones and Morgan, 1993); Shark Bay (Berggren, 1997b); Central Kimberleys: Rob Roy, Albert, East Montlivet, Wildcat reefs, Cassini, Jessieux, De Freycinet Is (Berggren, 1997c). Northern Territory: Cobourg Pen. (Bruce and Coombes, 1995). Queensland: Willis I.; Bet Reef; Restoration Rock; Heron I. and Wistari Reef, Capricorn Is (Patton, 1966); Heron I. (Bruce, 1981); Capricorn Is (Austin, Austin, and Sale, 1980).

\section{Further Distribution}

Type locality: Egypt. Reported from Egypt, Israel, Sudan, Eritrea, Djibouti, Yemen, Kenya, Zanzibar, Tanganyika, Moçambique, Madagascar, Seychelle Islands, Réunion, Mauritius, Maldive Islands, Chagos Islands, Sri Lanka, Andaman Islands, Singapore, Indonesia, Thailand, Vietnam, China, South China Sea, Japan, Philippine Islands, Papua New Guinea, Coral Sea, Marianas Islands, Marshall Islands, Fijian Islands, Society Islands, Tuamotu Islands, French Frigate Shoals, Johnson Atoll, Hawaiian Islands, and Easter Island.

\section{Genus Harpilius Dana, 1852}

\section{Harpilius bayeri Holthuis, 1981}

Periclimenes bayeri Holthuis, 1981: 792, fig. 3a-h. Davie, 2002: 324.

Harpilius bayeri. - Bruce, 2004: 5.

\section{Material examined}

Western Australia, Dampier Archipelago. WAM C 25911 (1 male, CL $2.7 \mathrm{~mm}$ ), stn DAl/98/13, Hammersley Shoal $\left(20^{\circ} 23.203^{\prime} \mathrm{S}, 116^{\circ} 46.691^{\prime} \mathrm{E}\right), 5.0$ m, coll. D. Heald, 21.10.1998.

\section{Host}

$$
\text { Pocillopora sp. [Scleractinia, Pocilloporidae]. }
$$

\section{Remarks}

Rostrum long and slender, dentition 7/3, tip well exceeding distal margin of scaphocerite. Fourth thoracic sternite with exceptionally long, slender, acute finger-like median process.

\section{Australian Distribution \\ Western Australia: Cartier Reef (Bruce, 1992).}

\section{Further Distribution}

Type locality: Ine Village, Arno Atoll, Marshall Islands. Known from Marshall Islands and Western Australia only.

\section{Genus Kemponia Bruce, 2004}

\section{Kemponia amymone (De Man, 1902) Figure 1B}

Periclimenes amymone De Man, 1902: 829, pl. 25 fig. 53. - Davie, 2002: 323

Kemponia amymone. - Bruce, 2004: 11.

\section{Material examined}

Western Australia, Dampier Archipelago. (1) WAM C 26634 (1 male, 1 juv.), stn DA3/99/36, Malus I. $\left(20^{\circ} 30.050^{\prime} \mathrm{S}, 116^{\circ} 40.594^{\prime} \mathrm{E}\right), 6.0-14.0 \mathrm{~m}$, 27.08.1999; (2) WAM C 25818 (1 male), stn DA1/98/ 03, Dolphin I. (20²4.320'S, $\left.116^{\circ} 56.108^{\prime} \mathrm{E}\right), 2.0-15.0$ m, 17.10.1998; (3) WAM C 25759 (1 ovig. female), stn DA1/98/01, Dolphin I. $\left(20^{\circ} 25.852^{\prime} \mathrm{S}\right.$, 116 52.953'E), 3.0-6.5 m, 17.10.1998; (4) WAM C 29300 (3 spms), stn DA3/99/36, Malus I. (20³0.050'S, $\left.116^{\circ} 40.594^{\prime} \mathrm{E}\right), 6.0-14.0 \mathrm{~m}, 27.08 .1999$; (5) WAM C 25861 (1 ovig. female), stn DA1/98/08, Angel I. $\left(20^{\circ} 29.180^{\prime} \mathrm{S}, 116^{\circ} 47.711^{\prime} \mathrm{E}\right), 2.0-8.0 \mathrm{~m}$, 20.10.1998; (6) WAM C 25879 (1 male, 4 ovig. females), stn DA1/98/06, Haüy I. (20²5.725'S, 116 $\left.57.580^{\prime} \mathrm{E}\right), 0.5-2.0 \mathrm{~m}, 19.10 .1998$; (7) WAM C 25863 (7 juvs), stn DA1/98/06, Haüy I. (20²5.725'S, $\left.116^{\circ} 57.580^{\prime} \mathrm{E}\right), 0.5-2.0 \mathrm{~m}, 19.10 .1998$; (8) WAM C 25767 (4 ovig. females), stn DA1/98/01, Dolphin I. (20²5.852'S, $\left.116^{\circ} 52.953^{\prime} \mathrm{E}\right), 3.0-6.5 \mathrm{~m}, 17.10 .1998$; (9) WAM C 25675 (2 males), stn DA1/98/29, Legendre I. $\left(20^{\circ} 24.566^{\prime} \mathrm{S}, 116^{\circ} 53.714^{\prime} \mathrm{E}\right), 4.5 \mathrm{~m}$, coll M. Hewitt, 27.10.1998.

\section{Host}

Generally Pocillopora spp, including $P$. damicornis [Scleractinia, Pocilloporidae], but (1) (2) were on Acropora sp. [Scleractinia, Acroporidae].

\section{Remarks}

The specimens present no special features. The dactyl of the third ambulatory pereiopod (Figure 1B) of specimen (3) is illustrated to show the distoventral spinulation of the propod, with a single slender spine only. The propod is ca 8.5 times longer than wide, 4.1 times longer than dactyl. The rostral dentition is $1+6-7 / 2-3$.

\section{Australian Distribution}

Western Australia: Quobba (Jones, 1990); Shark Bay (Berggren, 1997b); central Kimberleys, Rob Roy, Wildcat, Gibbings reefs, Lamarck I., Macleay, East Montlivet, Cassini, De Freycinet, Jesseux, Maret Is (Berggren, 1997c). Northern Territory: Darwin, East 


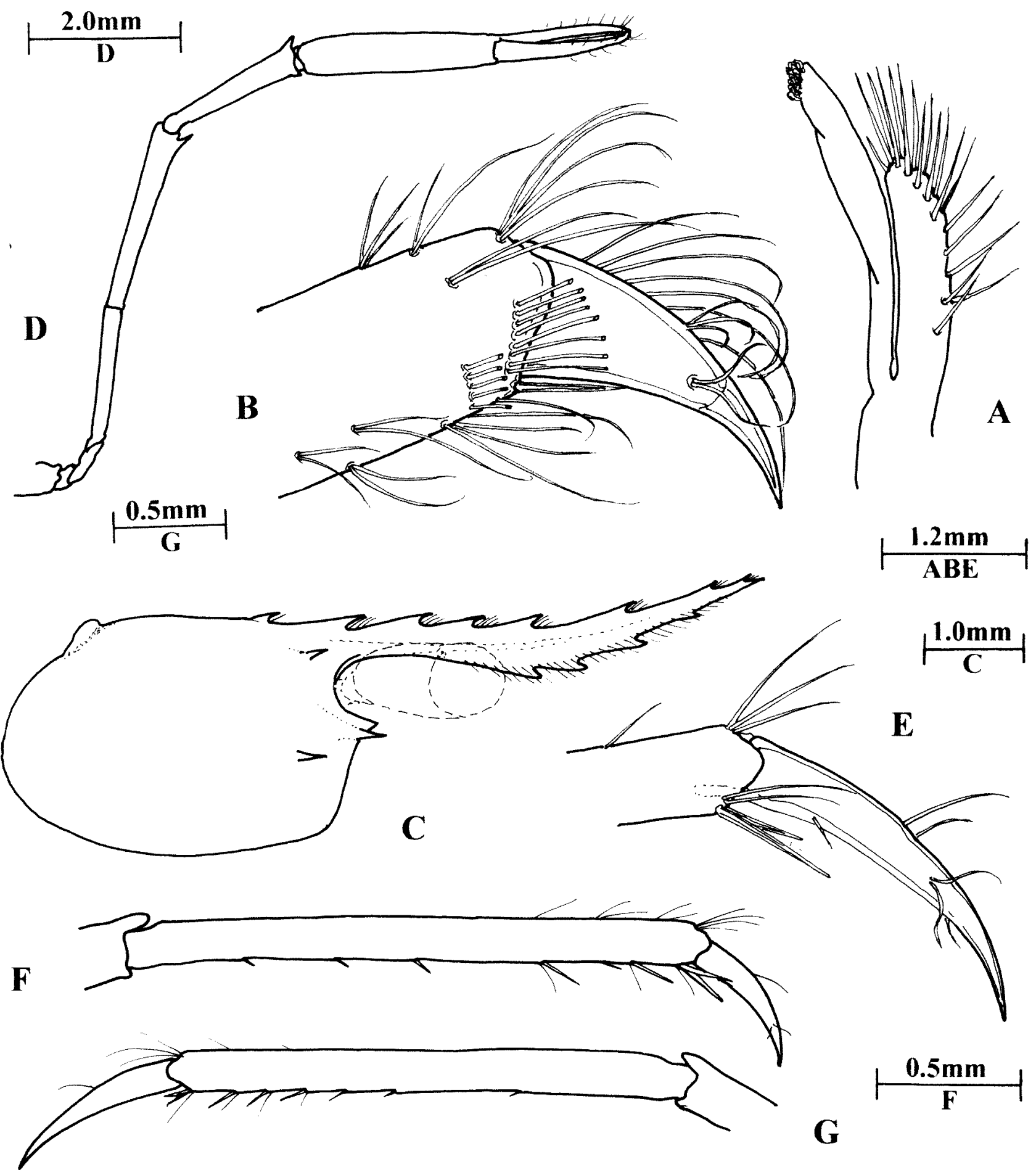

Figure 1 A, Harpiliopsis beaupresii (Audouin), male, WAM C 25879, second pleopod, appendices interna and masculina. B, Kemponia amymone (De Man), WAM C 25759, third pereiopod, distal propod and dactyl. C, Kemponia andamanensis (Kemp), WAM C 29309, carapace and rostrum. D, second pereiopod. E, third pereiopod, distal propod and dactyl. F, Kemponia grandis (Stimpson), WAM C 28096, third pereiopod, propod and dactyl. G, Kemponia aff. grandis, WAM C 28056, third pereiopod, propod and dactyl.

Pt (Bruce, 1983b, 1988a); Cobourg Peninsula (Bruce and Coombes, 1995). Queensland: Bet Reef, Restoration Rock; Heron I. and Wistari Reef, Capricorn Is; Myore, Moreton Bay (Patton, 1966); Heron I. (Bruce, 1981); One Tree I. (Bruce, 1977a); Orpheus and Lodestone (?) Is, Palm Is (Vytopil and Willis, 2001).

\section{Further Distribution}

Type locality: Ternate, Indonesia. Also known from the Nicobar Islands, Singapore, Vietnam, China, Indonesia, Papua New Guinea, Solomon Islands (?), Phillipines, and New Caledonia. 
Kemponia andamanensis Kemp, 1922 Figures $1 \mathrm{C}, \mathrm{D}, \mathrm{E}$

Periclimenes (Ancylocaris) andamanensis Kemp, 1922: 204, figs. 54-57.

Periclimenes andamanensis. - Davie, 2002: 323.

Kemponia andamanensis. - Bruce, 2004: 12.

\section{Material examined}

Western Australia, Dampier Archipelago: WAM C 29309 (1 male), stn DA2/99/75, off Goodwyn I. $\left(20^{\circ} 32.16^{\prime} \mathrm{S}, 116^{\circ} 33.70^{\prime} \mathrm{E}\right.$ to $\left.20^{\circ} 31.70^{\prime} \mathrm{S}, 116^{\circ} 33.20^{\prime} \mathrm{E}\right)$, rake box dredge, $19.0-14.0 \mathrm{~m}, 25.07 .1999$.

\section{Remarks}

Rostrum (Figure 1C) ca 1.8 times CL, slender upcurved, dentition $1+7 / 4$, with submarginal plumose setae ventrally, carapace with supraorbital spine well developed. Second pereiopods (Figure 1D) subequal, chela ca 1.8 times $\mathrm{CL}$, fingers feebly armed, carpus shorter than merus, with well developed medial tooth distally. The third pereiopod dactylus (Figure $1 \mathrm{E}$ ) is ca 6.5 times longer than its basal width, as compared with 7.5-8.0 times in the type material (Kemp, 1922). The specimen corresponds well with Kemp's figure of a syntype, except that the rostrum is relatively longer. In Kemp's specimen it appears ca subequal to the post-orbital carapace length. In his description Kemp states that there are 2-3 ventral rostral teeth, but in his key he indicates $2-4$, as in the present specimen. The material reported upon by $\mathrm{Li}$ (1996) from the Nansha Is had a shorter rostrum, as in Kemp's specimens, but also had a small supraorbital spine, as in the type of $P$. suvadivensis Borradaile (Bruce, 1978b).

This is a new record for Western Australia.

\section{Australian Distribution}

Queensland: Morton Bay (Wadley, 1978; Young and Wadley, 1979).

\section{Further Distribution}

Type locality: Port Blair, Andaman Islands. Known from Madagascar, Andaman Islands, China, South China Sea, Japan and Philippines.

\section{Kemponia elegans (Paul'son, 1875)}

Anchistia elegans Paul'son, 1875: 113, pl. 17, fig. 1.

Periclimenes (Ancylocaris) elegans. - Kemp, 1922: 215 , figs $60-62$.

Periclimenes elegans. - Li, 2000: 178, fig. 225. Davie, 2002: 326

Kemponia elegans. - Bruce, 2004: 14.

\section{Material examined}

Western Australia, Dampier Archipelago. WAM
C 29306 (1 ovig. female), stn DA2/99/18, off Sloping Pt, Burrup Pen. $\left(20^{\circ} 35.67^{\prime} \mathrm{S}, 116^{\circ} 54.97^{\prime} \mathrm{E}\right.$ to $20936.12^{\prime} \mathrm{S}, 116^{\circ} 55.09^{\prime} \mathrm{E}$ ), rake box dredge, 10.0-10.5 $\mathrm{m}, 16.07 .1999$.

\section{Remarks}

CL $4.3 \mathrm{~mm} .1+7 / 4$.

\section{Australian Distribution}

Western Australia: Hibernia Reef (Bruce, 1992); Central Kimberleys: Cassini, East Montlivet, Lamarck, White Is (Berggren, 1997c). Northern Territory: East Pt, Darwin (Bruce, 1988a); Orontes Reef; Port Essington; Cobourg Pen.; Oxley I. (Bruce and Coombes, 1995); Channel I., Darwin Harbour (Bruce and Coombes 1997). Queensland: Northwest Islet (McNeill, 1926): Diamond Islets; West Cay, Swain Reefs, (Bruce, 1977a); Heron I., Capricorn Is (Bruce, 1981f); Mourilyan Harbour (Hoedt et al., 2000).

\section{Further Distribution}

Type locality: Red Sea. Also known from Egypt, Sa'udi Arabia, Koweit, Aden, Kenya, Zanzibar, Tanganyika, Madagascar, Seychelle Islands, Minikoi, Pakistan (?), India, Sri Lanka, Andaman Islands, Nicobar Islands, Singapore, Indonesia, China, Hong Kong, Ryukyu Islands, Japan, Philippines, Papua New Guinea, Solomon Islands, Caroline Islands, Marshall Islands, Society Islands and Tuamotu Islands.

\section{Kemponia grandis (Stimpson, 1860) Figure $1 \mathrm{~F}$}

Anchistia grandis Stimpson, 1860: 39.

Periclimenes grandis. - Borradaile, 1898: 382. - Li, 2000: 186, fig. 235. - Davie, 2002: 327.

Periclimenes (Ancylocaris) grandis. - Kemp, 1922: 210, figs 58-59, pl. 7 fig. 10.

Kemponia grandis. - Bruce, 2004: 16.

\section{Material examined}

Western Australia, Dampier Archipelago. (1) WAM C 29303 (1 female), stn DA3/99/68, Nelson Rocks (2027.998'S, $\left.116^{\circ} 39.707^{\prime} \mathrm{E}\right), 6.0-24.0 \mathrm{~m}$, 06.09.1999; (2) WAM C 28096 (1 male, 1 female), stn DA3/99/68, Nelson Rocks (20\%27.998's, 116 39.707'E), 6.0-24.0 m, 06.09.1999. [Figure 1F, P3 propod]; (3) WAM C 25648 (1 ovig. temale), stn DA1/98/11, Dolphin I. (2030.249'S, 116 49.335'E), intertidal, 21.10.1998; (4) WAM C 28065 (2 ovig. females), stm DA3/99/59, West Lewis I. (2033.947's, $116^{\circ} 38.334^{\prime} \mathrm{E}$ ), intertidal, 04.09.1999; (5) WAM C 28065 (1male, 2 juv. males), stn DA3/99/59, West Lewis I. (2033.947'S, $\left.116^{\circ} 38.334^{\prime} \mathrm{E}\right)$, intertidal, 04.09.1999; (6) WAM C 25645 (1 spm), stn DAl/98/ 
30, Burrup Pen. (20 31.586'S, 116 51.088'E), 1.0-10.0 m, 27.10.98; (7) WAM C 29302 (2 ovig. females), stn DA3/99/62, East Lewis I. (20³7.499'S, $\left.116^{\circ} 39.182^{\prime} \mathrm{E}\right)$, intertidal, 05.09.1999.

\section{Remarks}

The specimens present no special features and had rostral dentitions of $1+6-7 / 2-4$.

The two juvenile males (5), WAM C 28065, with Cls $2.2,1.7 \mathrm{~mm}$, had a rostral dentition of $1+7 / 3 ; 1+$ $7 / 2$. The second pereiopods had a very small distoventral meral tooth on the larger specimen and was without these teeth on the smaller. The propod and distal dactylus of the third pereiopod (Fig.1F) are illustrated for comparison with the following taxon. Both had well developed appendices masculinae. The larger male, $C L 3.3 \mathrm{~mm}$, had a rostral dentition of $1+6 / 3$.

\section{Australian Distribution}

Western Australia: Hibernia Reef (Bruce, 1992c); Caparelli I., southern Kimberleys (Davie and Short, 1995); Shark Bay (Berggren, 1997b); central Kimberleys, Hedley, Slate, Maret Is, East Montlivet (Berggren, 1997c). Queensland: Port Molle (?) (Miers, 1884); Magnetic I. (Bruce, 1977a). Northern Territory: Darwin (Bruce, 1987b); East Pt, Darwin (Bruce, 1988a); Trepang Bay; Port Essington; McCluer I., Cobourg Pen. (Bruce and Coombes, 1995); Bullocky Pt, Cameron Beach, Channel I., Nightcliffe, Dudley Pt, Lee Point, Shell I., Weed Reef, Darwin Harbour (Bruce and Coombes, 1997). Queensland: Abbot Point (Hoedt et al., 2000).

\section{Further Distribution}

Type locality: Oshima, Japan. Also known from Egypt, Israel, Jibuti, Yemen, Kenya, Zanzibar, Tanganyika, Moçambique, Comoro Islands, Madagascar, Seychelle Islands, Sri Lanka, Burma, Malaya, Singapore, Indonesia, Vietnam, China, Japan, Papua New Guinea, Japan, Caroline Islands, Marshall Islands, Fijian Islands, Tuvalu and Tuamotu Islands.

\section{Kemponia aff. grandis}

Figure 1G

\section{Material examined}

Western Australia, Dampier Archipelago: WAM C 280561 ovig. female, stn DA3/99/38, Malus Is (20³0.632'S, $\left.116^{\circ} 38.788^{\prime} \mathrm{E}\right)$, intertidal, 27.08.1999.

\section{Remarks}

The single example has a rostral dentition of $1+6 /$ 3. It differs slightly from the norm in that the third pereiopod (Figure $1 G$ ) has a relatively longer dactyl, ca 0.36 of the propod length, as opposed to 0.24 , and the propod is more heavily spinulate distally, with a pair of distoventral spines and 5 spines on the distal half of the ventral margin, as opposed to a distoventral pair and 3 rather longer spines (cf. Figure 1F).

\section{Genus Palaemonella Dana, 1852}

\section{Palaemonella pottsi (Borradaile, 1915)}

Periclimenes (Falciger) pottsi Borradaile, 1915: 212.

Palaemonella pottsi. - Kemp, 1922: 126. - Bruce, 1970: 279, figs. 1, 3-7, pl. 1a-d. - Li, 2000: 103, fig. 113. - Davie, 2002: 316.

\section{Material examined}

Western Australia, Dampier Archipelago. (1) WAM C 25873 (1 male), stn DA1/98/10, Angel I. (2028.410'S, $\left.116^{\circ} 48.480^{\prime} \mathrm{E}\right), 0.0-2.0 \mathrm{~m}, 20.10 .1998$; (2) WAM C 28070, 2 juvs., stn DA3/99/47, Kendrew I. $\left(20^{\circ} 28.936^{\prime} \mathrm{S}, 116^{\circ} 32.519^{\prime} \mathrm{E}\right), 4.0-5.0 \mathrm{~m}$, coll. $\mathrm{S}$. Morrison, 30.08.1999; (3) WAM C 25810 (1 male, $1+6 / 2$, CL $3.0 \mathrm{~mm})$, stn DA1/98/10, Angel I. $\left(20^{\circ} 28.410^{\prime} \mathrm{S}, 116^{\circ} 48.480^{\prime} \mathrm{E}\right), 0.0-2.0 \mathrm{~m}, 20.10 .1998 ;(4)$ WAM C 28093 (1 ovig. female, 3 juvs), stn DA3/99/ 68, Nelson Rocks $\left(20^{\circ} 27.998^{\prime} \mathrm{S}, 116^{\circ} 39.707^{\prime} \mathrm{E}\right), 6.5 \mathrm{~m}$, 07.09.1999; (5) WAM C 28052 (1 ovig. female), stn DA3/99/2 (20³0.632'S, $\left.116^{\circ} 38.788^{\prime} \mathrm{E}\right)$, Malus I., 2.0 3.5 m, 27.08.1999; (6) WAM C 28077 (1 male, 1 ovig. female), stn DA3/99/55, Enderby I. (20³5.152'S, $\left.116^{\circ} 35.631^{\prime} \mathrm{E}\right), 17.0 \mathrm{~m}, 02.09 .1999$; (7) WAM C 25802 (1 ovig. female), stn DA1/98/10, Angel $\mathrm{I}$. (20²8.410'S, $116^{\circ} 48.480^{\prime} \mathrm{E}$ ), 0.0-2.0 m, 20.10.1998; (8) WAM C 29205 (1 ovig. female), stn DA2/99/10, NE of Delambre I. $\left(20^{\circ} 23.97 \mathrm{~S}, 117^{\circ} 04.82^{\prime} \mathrm{E}\right.$ to $20^{\circ} 23.72^{\prime} \mathrm{S}$, $117^{\circ} 04.70^{\prime} \mathrm{E}$ ), rake box dredge, $38.0 \mathrm{~m}, 15.07 .1999$; (9) WAM C 25680 (1 male, 1 ovig. female), stn DA1/ 98/33, Angel I. $\left(20^{\circ} 27.965^{\prime} \mathrm{S}, 116^{\circ} 49.692^{\prime} \mathrm{E}\right), 1.0-8.0$ m, 29.10.1999.

\section{Hosts}

Oxycomanthus sp.(1); Comanthina variabilis (Bell, 1882), (5) (7) (9); Comanthus alternans (Carpenter, 1881), (6); Comatella maculata (Carpenter, 1888), (2) [Crinoidea]. All appear to represent new host records. Also known to associate with Comanthina schlegeli (Carpenter, 1881), Comanthus bennetti (J. Müller), C. parvicirrus (J. Müller), C. timorensis (J. Müller), (Bruce, 1982b); Comantheria aff. rotula A.H. Clark, Himerometra robustipinna (Carpenter), (Bruce, 1983c); Stephanometra oxyacantha, Comanthus briareus (Bruce and Coombes, 1995) [Echinodermata, Crinoidea].

\section{Remarks}

The larger specimen (2), CL $1.4 \mathrm{~mm}$, from $\operatorname{Stn} 47$, had a rostral dentition of $1+6 / 1$, with the slender rostrum reaching to the end of the antennular peduncle, all rostral teeth are well developed but 
the first tooth on the carapace is noticeably smaller; the post-orbital ridges are very well marked but lack a distinct tubercle; the hepatic spine is very small. The mandible has a small, single segmented palp and the third pereiopod propod has short spines distally. The specimen is male and has a well developed, spiny appendix masculina on the second pleopod which clearly exceeds the endopod. The female (7) has a $C L$ of $4.8 \mathrm{~mm}$. The rostral dentition is $1+6-7 / 2$. Specimen (5) lacks second pereiopods.

\section{Australian Distribution}

Western Australia: southern Kimberleys, Leonie I., (Davie and Short, 1995); Shark Bay (Berggren, 1997b): central Kimberleys, Albert, Jamieson, Gibbings reefs, Cassini I., (Berggren, 1997c). Northern Territory: East Pt, Darwin (Bruce, 1988a); Cobourg Pen. (Bruce and Coombes, 1995). Queensland: Mabuaig and Murray (Borradaile, 1915; Potts, 1915); One Tree I. (Bruce, 1970; Bruce, 1977a); Heron I. (Bruce, 1981).

\section{Further Distribution}

Type locality: Murray Island, Torres Strait, Queensland. Reported from Zanzibar, Maldive Islands, Singapore, Indonesia, China, Japan, Philippine Islands, Papua New Guinea; Marshall Islands and New Caledonia.

\section{Palaemonella rotumana (Borradaile, 1898)}

Periclimenes rotumanus Borradaile, 1898: 383.

Palaemonella vestigialis. - Kemp, 1922: 123, figs 12, pl. 3 fig. 2. - Bruce, 1970: 276, pl. 1 e-f. - Li, 2000: 105, fig. 115. - Davie, 2002: 317.

\section{Material examined}

Western Australia, Dampier Archipelago. (1) WAM C 25904 (1 male), stn DA2/99/62, Flying Foam Passage, off Angel I. (20 $30.69^{\prime} \mathrm{S}, 116^{\circ} 48.58^{\prime} \mathrm{E}$ to $\left.20^{\circ} 31.17^{\prime} \mathrm{S}, 116^{\circ} 48.33^{\prime} \mathrm{E}\right)$, rake box dredge, $7.0-9.0$ m, 22.07.1999; (2) WAM C 29211 (1 male, 1 female, 1+6/2, 1 v. damaged spm, NWK), stn DA2/99/36, off High Pt, West Lewis I. (20 33.58'S, $116^{\circ} 36.87^{\prime} \mathrm{E}$ to $\left.20^{\circ} 33.88^{\prime} \mathrm{S}, 116^{\circ} 36.25^{\prime} \mathrm{E}\right)$, rake box dredge, $13.0 \mathrm{~m}$, 19.07.1999; (3) WAM C 29299 (ex WAM C 25692), (1 male, 1 ovig. female), stn DA1/98/31, Searipple Passage (20³1.230'S, $\left.116^{\circ} 51.182^{\prime} \mathrm{E}\right)$, intertidal, 28.10. 1998; (4) WAM C 25665 (1 ovig. female), stn DA1/ 98/08, Angel I. (20'29.180'S, 116 47.711'E), 2.0-8.0 m, 20.10.1998; (5) WAM C 25419 (1 ?female), stn DA3/99/64, West Lewis I. (20\%36.658's, $\left.116^{\circ} 36.956 \mathrm{E}\right), 2.0-5.0 \mathrm{~m}, 06.09 .1999$.

\section{Remarks}

The specimens of this well known species, several of which lacked second pereiopods, present no special features. The rostral dentition is $1+6-7 / 2-3$. Largest male, CL $4.5 \mathrm{~mm}$. Specimen (5) is juvenile, with poorly developed supraorbital ridges:

\section{Australian Distribution}

Western Australia: Rottnest I. (Black and Prince, 1983); Shark Bay (Jones, 1990); Cartier and Hibernia Reefs (Bruce, 1992); Rottnest I. (Jones and Morgan, 1993); Sunday I.; Montgomery Reef; Whirlpool Pass, southern Kimberleys (Davie and Short, 1995); central Kimberleys, Wildcat, Gibbings reefs, Hedley I. (Berggren, 1997c). Northern Territory: Darwin Harbour (Bruce, 1983b); Weed Reef, Darwin Harbour (Bruce, 1987d); East Pt, Darwin (Bruce, 1988a); Burford 1.; Port Bremer, Port Essington; Oxley I.; New Year I., Cobourg Pen. (Bruce and Coombes, 1995). Queensland: Moreton Bay; Low Is (Bruce, 1970); Herald Is, North East Cay; One Tree I. (Bruce, 1977a); Heron I. (Bruce, 1981); John Brewer Reef (Bruce, 1987d).

\section{Further Distribution}

Type locality: Rotuma Island, Fijian Islands. Reported from Israel (Haifa), Egypt, Suez, Yemen, Kenya, Zanzibar, Tanganyika, Moçambique, Madagascar, Comoro Islands, Seychelle Islands, Maldive Islands, Sri Lanka, Andaman Islands, Nicobar Islands, Burma, Malaya, Singapore, Vietnam, China, Hong Kong, South China Sea, Ryukyu Islands, Japan, Indonesia, Papua New Guinea, Philippines, New Caledonia, Marshall Islands, Mariannas Islands, Fijian Islands, and Hawaii(?). To depths of $120 \mathrm{~m}$. Now also occurring in the eastern Mediterranean Sea.

\section{Palaemonella spinulata Yokoya, 1936 Figures 2, 3}

Palaemonella spinulata Yokoya, 1936: 135, fig. 4. Li, 2000: 106. - Davie, 2002: 317.

Not Palaemonella spinulata. - Bruce, 1975: 177, figs 6-7. - Li, 2000: fig. 114.

\section{Material examined}

Western Australia, Dampier Archipelago. (1) WAM C 29308 (1 ovig. female), stn DA2/99/73, off Rocky Head, Enderby I. $\left(20^{\circ} 40.14^{\prime} S, 116^{\circ} 27.69^{\prime} \mathrm{E}\right.$ to $\left.20^{\circ} 39.93^{\prime} \mathrm{S}, 116^{\circ} 27.96 \mathrm{E}\right)$, rake box dredge, $12.5 \mathrm{~m}$, 24.07. 1999; (2) WAM C 28094 (1 male, 1+ 7/2, CL $3.2 \mathrm{~mm}$, drawn), stn DA3/99/68, Nelson Rocks (20²7.998'S, 116 39.707' E), 6.5 m, 07.07.1999.

\section{Diagnosis}

Rostrum (Figure 2A) exceeding antennular peduncle, slightly upturned. Orbit obsolete; supraorbital spines present, small, ridges absent; hepatic spine larger; inferior orbital angle (Figure $2 B$ ) produced, acute; exceeded by antennal spine; $R$ 
1+7/2, ca 1.1 times CL. pleuron 4 (Figure 2l) bluntly rounded posteroventrally, pleuron 5 feebly acute. Mandibular palp 2-segmented; second maxilliped with rudimentary podobranch. Second pereiopod (Figure 2F) with distoventral meral tooth, without ischial teeth; carpus (Figures 2G) with short blunt distolateral tooth. Third pereiopod dactyl (Figure $3 \mathrm{C}$ ) ca 0.27 of propod length (Figure $2 \mathrm{H}$ ); dactyls $\mathrm{ca}$ 5 times longer than wide, ventral margin concave; propods ca 12 times longer than wide. Appendix masculina subequal to endopod length. Dorsal telson spines at 0.33 and 0.66 of length; posterior margin with acute median point.

\section{Type}

The holotype specimen from Misaki is considered to be lost ( Holthuis, 1952; Bruce, 1970; Okuno, pers. comm.). The designation of a neotype would appear useful but one from Japanese or nearby waters would be more appropriate than one of the present specimens.

\section{Remarks}

(1) Supraorbital spine well developed, mandibular palp 2-segmented, CL $2.5 \mathrm{~mm}, 1+6 / 2$. (2) large spm, CL $3.2 \mathrm{~mm}$, with relatively small supraorbital spines; rostrum exceeding scaphocerite, tip acute, slightly deflected. The second pereiopod is robust, the chela ca 2.1 times $\mathrm{CL}$. The appendix masculina is slightly longer than endopod, with numerous spines, twice length of the appendix interna and the endopod.

The specimens referred to $P$. spinulata Yokoya by Bruce (1975) from Tanganyika and Kenya are now not considered to belong to this species. The taxon has now been provided with a new name (Bruce, 2002a).

This is a new record for Western Australia.

\section{Australian Distribution}

Queensland: Heron I. (Bruce, 1981); Morton Bay, Dunwich (Bruce, 1983). Northern Territory: Cobourg Peninsula (Bruce and Coombes, 1995); Darwin Harbour, Fannie Bay (Bruce and Coombes, 1997).

\section{Further Distribution}

Type locality: Misaki, Japan. Reported only from La Réunion, China and Japan.

\section{Palaemonella sp.}

\section{Material examined}

Western Australia, Dampier Archipelago. WAM

C 25659 (1 female), stn DA1/98/34, Tozer I. $\left(20^{\circ} 27.684^{\prime} \mathrm{S}, 116^{\circ} 50.486^{\prime} \mathrm{E}\right)$, intertidal, 29.10 .1998 .

\section{Remarks}

The specimen, which lacks both second pereiopods, has a 2 -segmented palp. The rostral dentition is $2+6 / 3$, the rostrum ca 1.1 of $C L$, not exceeding the scaphocerite, and the supraorbital carina and tubercle, as in $P$. pottsi and $P$. rotumana, is obsolete. The posteroventral angle of the fourth pleuron is rounded, the fifth acute. The third ambulatory pereiopod has a slender propod, ca 15 times longer than wide, with a pair of unequal distoventral spines and 4 isolated spines on the distal half of the ventral border, with the dactyl ca 0.26 of the propod length, 6.5 times longer than its basal width, smoothly concave ventrally.

The specimen shows some resemblance to Palaemonella foresti, but the dactyls of the ambulatory pereiopods are much longer and more slender, compared with 0.18 of the propod length and 3.6 times longer than the basal depth in $P$. foresti (Bruce, 2002b).

Specimens of Palaemonella aff. rotumana have been reported from the Seychelle Is and from Kenya which lack a tuberculate supraorbital ridge (Bruce, 1974a; 1976; 2002b).

\section{Genus Periclimenella Duris and Bruce, 1995}

\section{Periclimenella spinifera (De Man, 1902)}

Periclimenes Petithouarsi var. spinifera De Man, 1902: 284.

Periclimenes (Ancylocaris) spiniferus. - Kemp, 1922: 195.

Periclimenes (Harpilius) spiniferus. - Holthuis, 1952: 76, fig. 30.

Periclimenella spinifera. - Duris and Bruce, 1995 , 656, figs 19-20. - Li, 2000:144, fig. 178. - Davie, 2002: 321.

\section{Material examined}

Western Australia, Dampier Archipelago. WAM C 28087 (2 ovig. females), stn DA3/99/64, West Lewis I. $\left(20^{\circ} 36.658^{\prime} \mathrm{S}, 116^{\circ} 36.956^{\prime} \mathrm{E}\right), 2.0-5.0 \mathrm{~m}$, 06.09.1999.

\section{Remarks}

The specimens present no special features. This species is usually abundant on coral reefs and it is surprising that so few specimens were collected from the Dampier Archipelago.

\section{Australian Distribution}

Western Australia: Cartier and Hibernia Reef (Bruce, 1992c); Sunday I., southern Kimberleys (Davie and Short, 1995, as Periclimenes); central Kimberleys: Montgomery reef, Maret Is (Berggren, 1997c). Northern Territory: Dudley Pt, Darwin (Bruce, 1983); Weed Reef, Darwin Harbour (Bruce, 

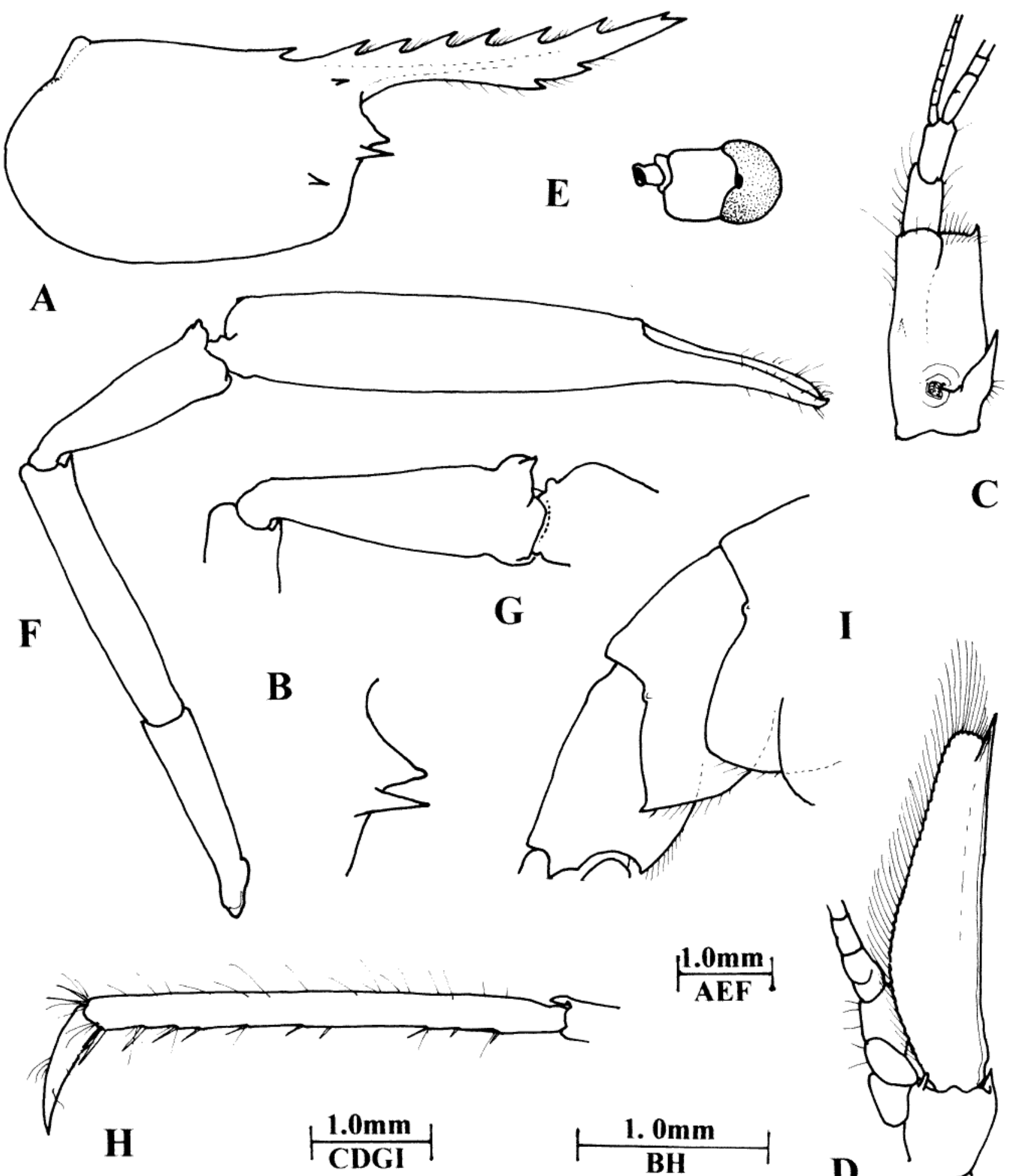

C

Figure 2 Palaemonella spinulata Yokoya, male, WAM C 28094. A, carapace and rostrum. B, inferior orbital region. C, antennule. D, antenna. E, eye. F, second pereiopod. G, same, carpus. H, third pereiopod, propod and dactyl. I, abdomen, fourth and fifth pleura.

1987b, as P. spinifer); East Pt, Darwin (Bruce, 1988a); Cobourg Pen. (Bruce and Coombes, 1995); East Pt; Channel I., Darwin Harbour (Bruce and Coombes 1997). Queensland: Northwest I. (McNeill, 1926); Low I. (Stephenson, et al., 1931); Low Is (McNeill, 1968); Great Barrier Reef (Patton, 1966); Heron I. (Patton, 1974; Bruce, 1981); One Tree
I. (Austin, Austin and Sale, 1980); Lizard I. (Bruce, 1983).

\section{Further Distribution}

Type locality: Ternate, Ambon, Indonesia. Reported from Kenya, Tanganyika, Madagascar, Seychelle Islands, Réunion, Maldive Islands, 

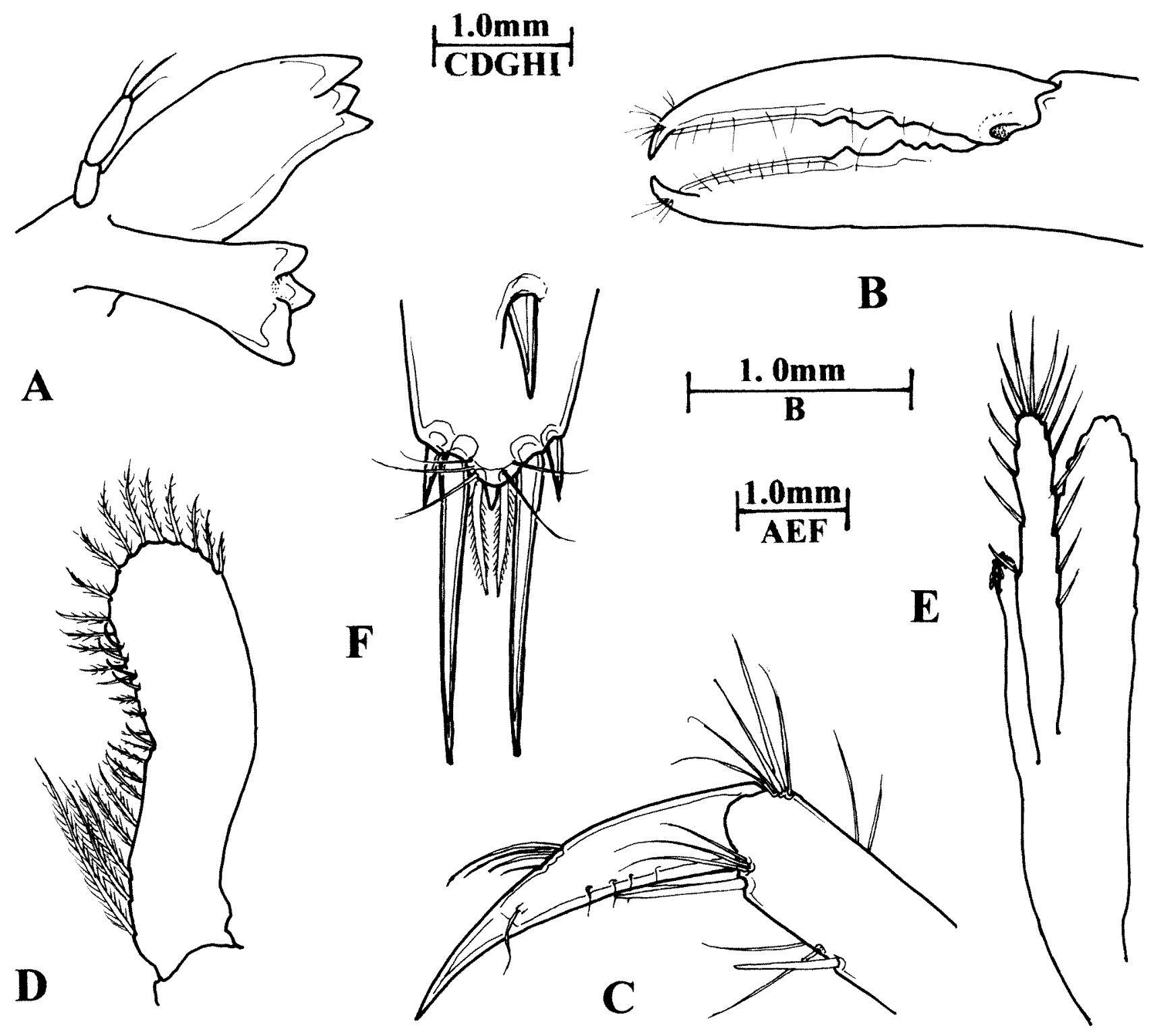

A

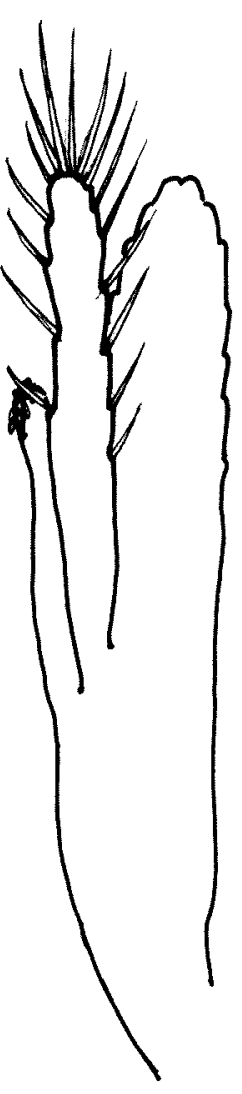

Figure 3 Palaemonella spinulata Yokoya, male, WAM C 28094. A, mandible. B, second pereiopod chela, fingers. C, third ambulatory pereiopod, distal propod and dactyl. D, first pleopod, endopod. E, second pleopod, endopod. F, posterior telson, dorsal spine inset.

Chagos Islands, Gulf of Manaar, Andaman Islands, Nicobar Islands, Burma, Malaya, Singapore, Vietnam, Indonesia, China, South China Sea, Japan, Philippines, Papua New Guinea, Mariannas Islands, Marshall Islands, Fijian Islands, Samoan Islands and Society Islands.

\section{Genus Periclimenaeus Borradaile, 1915}

\section{Periclimenaeus arabicus (Calman, 1939)}

Periclimenes (Periclimenaeus) arabicus Calman, 1939: 210, fig. 4.

Periclimenaeus arabicus. - Holthuis, 1952: 13, 130. Li, 2000: 116, fig. 127. - Davie, 2002: 318.
Periclimenaeus ohshimai Miyake and Fujino, 1967: 275, fig. 1.

\section{Material examined}

Western Australia, Dampier Archipelago. WAM C 28049 (1 ovig. female), stn DA3/99/36, Malus I. $\left(20^{\circ} 33.58^{\prime} \mathrm{S}, 116^{\circ} 36.87^{\prime} \mathrm{E}\right.$ to $\left.20^{\circ} 33.88^{\prime} \mathrm{S}, 116^{\circ} 36.25^{\prime} \mathrm{E}\right)$, $6.0-14.0 \mathrm{~m}, 27.08 .1999$.

\section{Remarks}

Rostrum not exceeding eyes, dentition $6 / 1$, acute supraorbital tubercle distinct. CL $2.0 \mathrm{~mm}$. Noted as collected from Pocillopora, but presumably from an encrusting sponge host.

This is a new record for Western Australia. 


\section{Australian Distribution}

Reported from central Kimberleys by Berggren (1997c). Queensland: Heron 1. (Bruce, 1981; 1983) Northern Territory: Cobourg Pen. (Bruce and Coombes, 1995).

\section{Further Distribution}

Type locality: Oman: 19²2.6 N. 57 53.0'E., $13.5 \mathrm{~m}$. Reported from Oman, "Cotes d'Arabie", Djibuti, Kenya, Zanzibar, Tanganyika, Seychelle Islands, Maldive Islands, Vietnam, China, Hong Kong, Japan, New Caledonia and Fijian Islands.

\section{Periclimenaeus hecate (Nobili, 1904)}

Coralliocaris hecate Nobili, 1904: 232. - Nobili, 1906: 58 , pl. 3 , fig. 2.

Periclimenaeus tridentatus. - Holthuis, 1952: 145 (part.).

Periclimenaeus hecate. - Bruce, 1974: 1574, figs 1112, 13e. - Li, 2000: 124, fig. 143. - Davie, 2002: 320. - Marin et al., 2005: 205, fig. 5a-h.

\section{Material examined}

Western Australia, Dampier Archipelago. WAM C 25906 (1 ovig. female), stn DA1/98/32, Legendre I. (20²3.520'S, $\left.116^{\circ} 54.110^{\prime} \mathrm{E}\right), 5.0-17.0 \mathrm{~m}, 28.10 .1998$.

\section{Remarks}

Rostrum short, not exceeding eyes, dentition 3/0. The major second pereiopod and all ambulatory pereiopods except one fifth pereiopod are lacking so the determination cannot be considered certain. The fifth pereiopod dactyl lacks a distal accessory spine, but a minute proximal ventral styliform tooth may be present. The minor second pereiopod dactyl has ca 40 small acute teeth, of diminishing size proximally, palm sparsely setose. CL $2.5 \mathrm{~mm}$.

\section{Australian Distribution}

Western Australia: C. Jaubert (Balss, 1921, as ?Coralliocaris); central Kimberleys, Albert Reef, Maret Is (Berggren, 1997c). Queensland: Heron I., Wistari Reef (Bruce, 1981; Bourdon and Bruce, 1983); One Tree I. (Austin, Austin and Sale, 1980).

\section{Further Distribution}

Type locality: Djibuti. Reported from Djibuti, Kenya, Comoro Islands, Seychelle Islands, Réunion, Maldive Islands, Indonesia, Vietnam and China.

\section{Periclimenes affinis (Zehntner, 1894)} Figure 4

Palaemonella affinis Zehntner, 1894: 208.

Periclimenes (Harpilius) affinis. - Holthuis, 1958: 6 , fig. 2.
Periclimenes affinis. - Bruce, 1980a: 2, figs. 1-3. Davie, 2002: 323

\section{Material examined}

Western Australia, Dampier Archipelago. (1) WAM C 29304 (2 males, 1 ovig. female), stn DA3/ 99/68, Nelson Rocks (20²7.998'S, 116 39.707'E), 6.5 m, 07.10.1999, (2) WAM C 29301 (23 [7 ovig. females]), DA3/99/55, Enderby I. (20³5.152'S, $116035.631 \mathrm{E}$ ), intertidal, 02.10.1999.

\section{Hosts}

(1) crinoid $Z$ 5953, Comanthina variabilis (Bell, 1882). (2) crinoid $Z$ 5557, Comanthina alternans (Carpenter, 1881)[Crinoidea]. Both hosts are new host records. Associations with Comatula cratera (H.L. Clark), Comanthus sp. (Bruce, 1982); Comanthina schlegeli (Carpenter, 1881) have been reported (Bruce, 1982c) [Echinodermata, Crinoidea].

\section{Remarks}

This represents a new record to the Western Australian fauna.

The mature specimens are as previously described (Holthuis, 1958; Bruce, 1980). A small juvenile specimen, CL $1.3 \mathrm{~mm}$, in (2), has the rostrum (Figure $4 \mathrm{~A}$ ) ca 0.75 of the $\mathrm{CL}$, dentition of $4 / 0$. The antennal spine is well developed, subcarinate, distinctly exceeding the inferior orbital angle. The hepatic spine, at a slightly lower level, is short and robust. The comea is large, hemispherical, ca 0.28 of the CL. The first and second pereiopods are very short, reaching only to $c a$ the distal end of the scaphocerite. The first pereiopod (Figure 4B) has a very short, robust carpus, slightly longer than the palm of the chela, which is subequal to the finger length. The fingers are unarmed, without distinct cutting edges. Second pereiopods (Figure 4C) are subequal, more slender, with the chela ca 0.8 of the $\mathrm{CL}$; the fingers are a little shorter than the palm and unarmed. The third pereiopod has the propod (Figure $4 \mathrm{D}$ ) equal to $\mathrm{ca} 0.5$ of the $\mathrm{CL}$, robust, with four slender spines ventrally, with numerous long filamentous setae distoventrally. The dactyl is ca 0.25 of the propod length, simple, with a slender curved unguis, ca 0.66 of the corpus length. The specimen is probably an early post larval juvenile.

\section{Australian Distribution}

Northern Territory: Black Point, Port Essington, Cobourg Peninsula (Bruce and Coombes, 1995). Queensland: Capricorn Is, Wistari Reef ((Bruce, 1981).

\section{Further Distribution}

Type locality: Ambon, Moloccan Islands, Indonesia. Known from Indonesia, China, South China Sea, Japan (?), Philippines, Papua New Guinea and New Caledonia. 

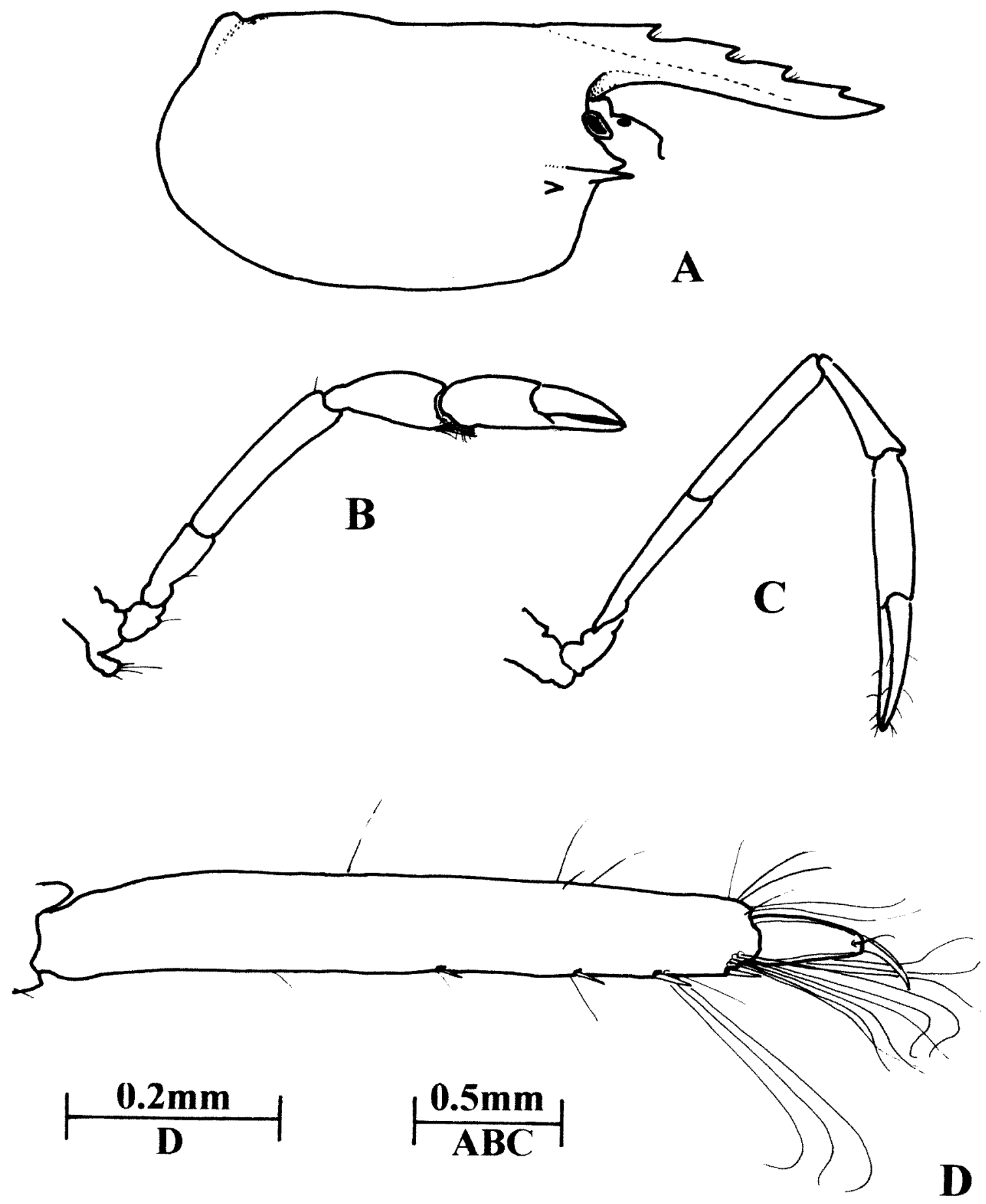

Figure. 4 Periclimenes affinis (Zehntner), juvenile, WAM C 29301. A, carapace and rostrum. B, first pereiopod. C, second pereiopod. $\mathbf{D}$, third pereiopod, propod and dactyl.

Genus Periclimenes Costa, 1844

Periclimenes alegrias Bruce, 1987

Periclimenes alegrias Bruce, 1987a: 143, figs 1A, 2 5, 15 A-C. - Li, 2000: 152, fig. 186. - Davie, 2002: 323.

\section{Material examined}

Western Australia, Dampier Archipelago. WAM C 29305 (1 ovig. female), stn DA3/99/68, Nelson Rocks (20²7.998'S, $\left.116^{\circ} 39.707^{\prime} \mathrm{E}\right), 6.5 \mathrm{~m}, 07.09 .1999$.

\section{Host}

Comanthina variabilis (Bell, 1882) [Echinodermata, Crinoidea].

\section{Remarks}

The single specimen, CL $2.1 \mathrm{~mm}$, agrees closely with the original description. It has a rostral dentition of $8 / 0$ and lacks the minor second pereiopod and all ambulatory pereiopods except the fourth right pereiopod.

Previously reported in association with 
Stephanometra spicata (Carpenter) and Lamprometra palmata (Müller). The entries in the table in Bruce (1986, p. 151) are incorrect.

\section{Australian Distribution}

Western Australia: Whirlpool Pass, southern Kimberleys (Davie and Short, 1995). Northern Territory: Coral Bay, Port Essington, Arnhem Land (Bruce, 1987a); East Point, Darwin (Bruce, 1988); West Vernon I. (Bruce, 1990a); Orontes Reef and Coral Bay, Cobourg Peninsula (Bruce and Coombes, 1995).

\section{Further Distribution}

Type locality: Coral Bay, Port Essington, Cobourg Peninsula, Northern Territory. Not known from outside Northern Territory waters. Davie (2002) cites WA record.

\section{Periclimenes burrup sp. nov.}

Figures 5-7

Periclimenes sinensis. - Bruce and Coombes, 1995: 133 , figs $12 \mathrm{~b}-\mathrm{c}$.

\section{Material examined}

Holotype: WAM C 25672 (ovig. female, CL 2.0; carapace and rostrum 3.8; total body length ca. 11.0; major second pereiopod chela 3.3; minor second pereiopod chela 2.2; length of ovum 0.5), stn DA1/ 98/30, Burrup Pen. $\left(20^{\circ} 31.59^{\prime} \mathrm{S}, 116^{\circ} 52.09^{\prime} \mathrm{E}\right), 11.0 \mathrm{~m}$, 27.10.1998.

Paratypes: WAM C 35561 (1 male), stn DA1/98/ 30, Burrup Pen. (20³1.59'S, $\left.116^{\circ} 52.09^{\prime} \mathrm{E}\right), 11.0 \mathrm{~m}$, 27.10.1998; WAM C 35562 (1 female), stn DA1/98/ 30, Burrup Pen. $\left(20^{\circ} 31.59^{\prime} \mathrm{S}, 116^{\circ} 52.09^{\prime} \mathrm{E}\right), 11.0 \mathrm{~m}$, 27.10.1998.

Western Australia, Dampier Archipelago. (1) WAM C 25672 (ca 44 spms, 29 ovig. females), stn DA1/98/30, Burrup Pen. (20³1.59'S, 116 52.09'E), $11.0 \mathrm{~m}, 27.10 .1998$; (2) C WAM (1 ovig. female), stn DA2/99/71, off Rocky Head, Enderby I. (2041.49'S, $116^{\circ} 28.05^{\prime} \mathrm{E}$ to $\left.20^{\circ} 41.55^{\prime} \mathrm{S}, 116^{\circ} 28.36^{\prime} \mathrm{E}\right)$, rake box dredge, 10.5 m, 24.7.99; (3) WAM C 26625 (1 female, 3 juvs), stn DA2/99/56, off Roly Rock (20 $30.10^{\prime} S$, $116^{\circ} 28.27^{\prime} \mathrm{E}$ to $\left.20^{\circ} 29.88^{\prime} \mathrm{S}, 116^{\circ} 27.93^{\prime} \mathrm{E}\right)$, rake box dredge, 33.0-34.5 m, 21.7.99.

\section{Host}

(1)(2) Dendronephthea sp. [Alcyonacea]. (3) unidentified sponge.

\section{Diagnosis}

A small Periclimenes of the obscurus group. Rostrum (Figure 5B) horizontal, slender, subequal to CL (Figure 5A), dentition 8-11/1-2, mainly 8-9/2 $(72 \%)$, first tooth on carapace, first 3 teeth semiarticulated, inferior orbital angle slightly produced, blunt, not reaching to end of antennal spine, corneal diameter (Figure 5F) 0.24 of CL, scaphocerite (Figure 5E) 2.27 times longer than wide, bluntly rounded distally, lateral tooth falling short of distal lamellar margin; mouthparts similar to $P$. toloensis (see Bruce, 1982, p. 262, fig. 17), third maxilliped (Figure 5G) slender, ischiomerus and basis feebly separated, with well developed rounded epipod and rudimentary arthrobranch, thoracic sternites (Figure 5H) armed, fourth with paired confluent subacute submedian processes, fifth with paired discrete blunt submedian processes, sixth with blunt median process, first pereiopod (Figure 6B) with chela (Figure 6C) subequal to carpus, coxa with strong ventral process (Figure 6D), second pereiopods unequal, robust, major chela (Figure 6E) with fingers (Figure 6F) 0.6 of palm length, feebly dentate (Figure 6G), fixed finger with three small teeth proximally, central tooth smaller, single tooth on dactylus, teeth slightly larger in male, carpus short, half palm length, chela 1.3 times CL, merus longer than palm, unarmed, third ambulatory dactyls slender, 0.25 of propod length, biunguiculate, unguis 0.57 of corpus length, accessory tooth slender, 0.5 of unguis length; propod with distoventral spine, 4 single ventral spines; telson (Figure 5I) ca 0.75 of CL, dorsal spines normal, at ca 0.5 and 0.75 of telson length, lateral posterior spines small, posterior margin (Figure 5J) bluntly angular, without acute median process, lateral spines ca 0.2 of intermediate spine length, intermediate spines (Figure 5K) 0.25 of telson length, robust, distally blunt, submedian spines (Figure 5K) 0.4 of intermediate spine length, distally acute, setulose; uropods without special features. Colouration unknown.

\section{Systematic position}

Periclimenes burrup is closely related to $P$. sinensis Bruce, 1969 (Bruce, 1969; 1982a). The new species is most readily distinguished by its stouter form, the much more robust unequal second pereiopods, with the chelae having relatively shorter fingers. The scaphocerite is broader, ca 2.7 times longer than wide, as opposed to ca 3.0 times, and distally rounded rather than angular. The unguis of the ambulatory dactyl is shorter in P. burrup, ca 0.57 of the corpus length, 0.7 in $P$. sinensis, with the accessory tooth more divergent. The poster margin of the telson has an acute median point in $P$. sinensis, not present in $P$. burrup. The condition of the thoracic sternites in $P$. sinensis is unknown. The fourth and fifth sternites are similar to $P$. terangeri, which is distinguished by the presence of a distinct epigastric spine, longer paired ventral spines on the ambulatory propods (Bruce, 1998).

\section{Etymology}

From Burrup, original name for locality of capture, used in apposition. 


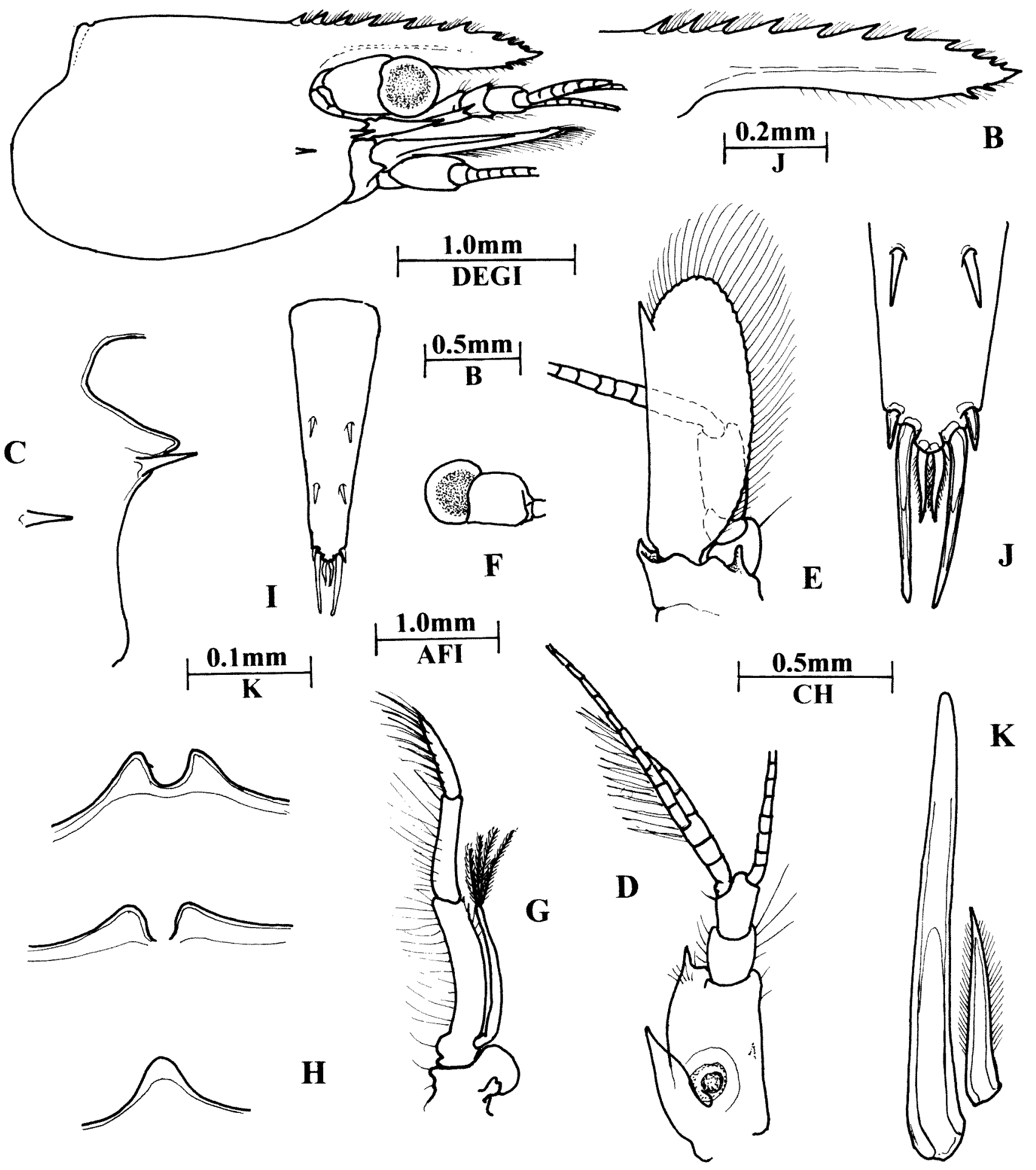

Figure 5 Perclimenes burrup sp. nov., ovigerous females, Burrup Pen., WAM C 25672. A, carapace and appendages. B, rostrum. C, carapace, orbital region, right lateral. D, antennule. E, antenna. F, eye. G, third maxilliped. $\mathbf{H}$, fourth to sixth thoracic sternites. I, telson. $\mathbf{J}$, same, posterior end. $\mathbf{K}$, same, submedian and intermediate posterior spines. AB, holotype (ovig. female) WAM C 25672. C-K, paratype (female) WAM C 35562.

\section{Remarks}

The closely related Periclimenes sinensis is reported from Hong Kong (type locality), Japan (as Periclimenes setoensis), Philippines and Northern Territory only. The second pereiopods of $P$. sinensis are reported to be subequal and similar, as are those of its synonym $P$. setoensis Fujino and Miyake, 1969. The $P$. sinensis material reported from the Northern Territory (Bruce and Coombes, 1995) should be referred to $P$. burrup on account of the asymmetry of the second pereiopods and the robust development of the major chela. Periclimenes 


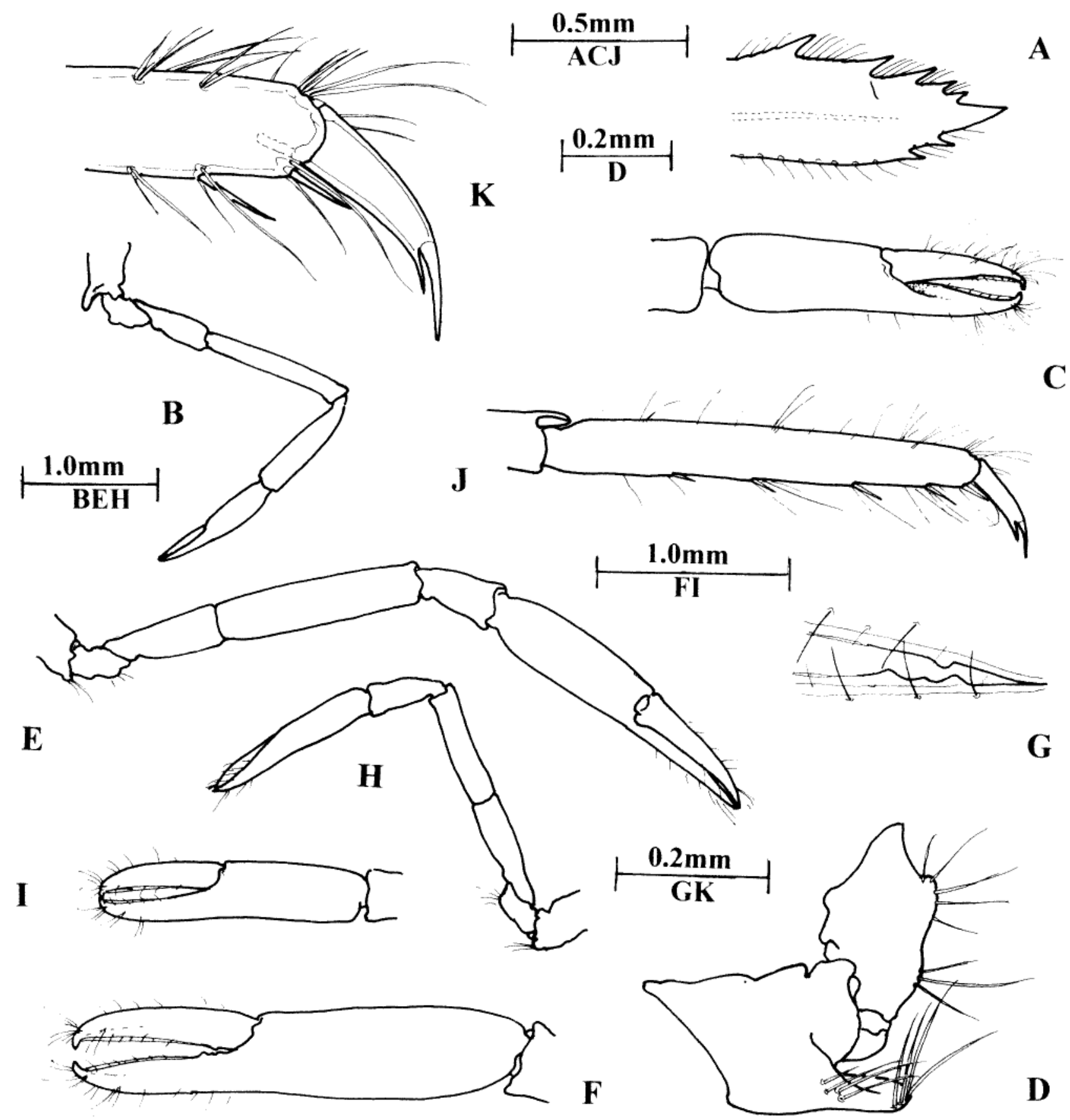

Figure 6 Perclimenes burrup sp. nov., ovigerous females, Burrup Pen., WAM C 25672. A, distal rostrum. B, first pereiopod. C, same, chela. D, same, basis and coxa. E, major second pereiopod. F, same, chela. G, same, proximal cutting edges of fingers. $\mathbf{H}$, minor second pereiopod. $\mathbf{I}$, same, chela. J, third pereiopod, propod and dactyl. K, same, distal propod and dactyl. A, holotype (ovig. female WAM C 25672. B-K, paratype (female) WAM C 35562.

sinensis is, therefore, not to be included in the Australian pontoniine fauna. Possibly also closely related is $P$. batei Holthuis. The type specimen was collected from the Philippines, off Sibago, $6^{\circ} 57^{\prime} \mathrm{N}$ $122^{\circ} 28^{\prime} \mathrm{E}, 7-45 \mathrm{~m}$, and described by Bate (1888) as Palaemonella orientalis. It was distinguished from that species and renamed Palaemonella batei by Borradaile (1917) and later transferred to the genus Periclimenes bo Holthuis (1959), as it lacked a mandibular palp. Its systematic position is obscure. It is small (CL $0.9 \mathrm{~mm}$ ), clearly juvenile, and as it may have been obtained from plankton, possibly only post-larval. It has six dorsal rostral teeth, all anterior to the orbital margin and is without an epigastric spine. The first pereiopod is distinctly different from $P$. burrup and $P$. sinensis as the carpus is ca 2.7 times longer than wide and subequal to the palm length. Similar proportions 

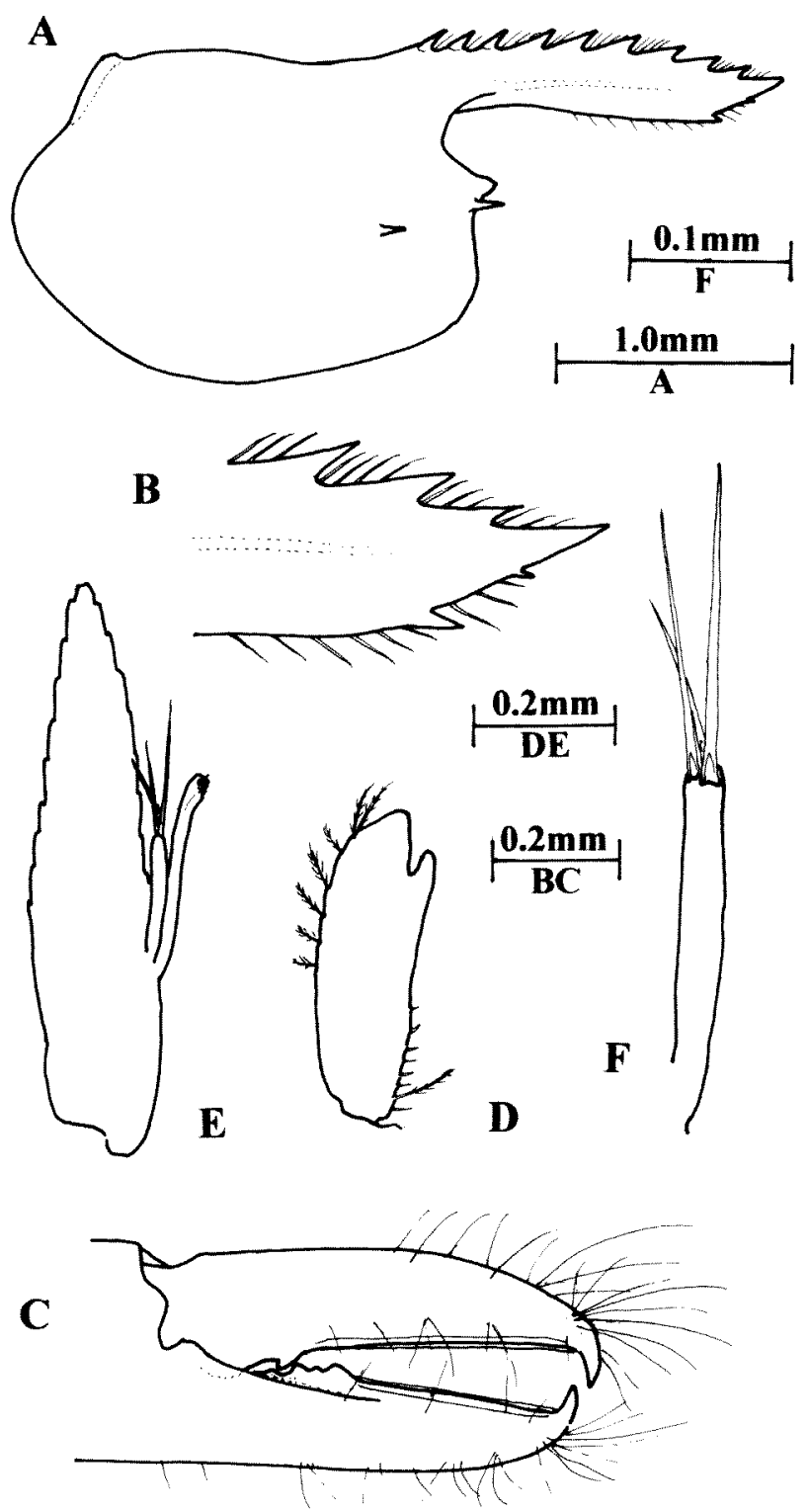

Figure 7 Perclimenes burrup sp. nov, male, paratype, Burrup Pen., holotype (ovig. female) WAM C 35561. A, carapace and rostrum. B, tip of rostrum. C, second pereiopod, fingers. $\mathbf{D}$, first pleopod, endopod. E, second pleopod, endopod. F, same, appendix masculina.

have been observed in juveniles of other species of Periclimenes in which the carpus is much longer in the adult. This applies also to the reduced rostral dentition. Periclimenes batei could be a juvenile of any species of the obscurus-group. Periclimenes batei has since been possibly recorded from the Seychelle Is ( $P$. aff. batei Fransen, 1994) and Western Australia (as P. ? batei Bruce, 1992). The type specimen of $P$. batei is preserved in the collection of the Natural History Museum, registration number BMNH 1888.22 (P. Clark, 21-02-03), and is incomplete: the body is mainly intact, somewhat shrivelled, with only one first pereiopod, one second pereiopod and one ambulatory pereiopod, the last two detached.

\section{A Key to the Obscurus Species Group}

1. Carapace with isolated epigastric spine ......... 2

Carapace without epigastric spine .................. 8

2. Ambulatory dactyls stout, with accessory tooth stouter than unguis; R. $2+7 / 2$

\section{$P$. incertus Borradaile}

Ambulatory dactyls slender, accessory spine more slender and shorter than unguis ........ 3

3. Second pereiopods subequal, similar, carpus subequal to palm, antennal spine postmarginal, inferior orbital angle feebly developed; R. 1+6-9/1-2 ... P. obscurus Kemp

Second pereiopods markedly unequal, carpus much shorter than palm

4. Rostral lamina deep, 13-17 dorsal teeth, 3-4 ventral .................... P. hongkongensis Bruce

Rostral lamina not deep, rostral teeth less than 11 dorsal, 3 ventral 5

5. Dorsal telson spines minute; major chela palm slender, ca 4.3 times longer than wide; inferior orbital angle strongly produced; $R$. $1+8 / 1$ P. toloensis Bruce

Dorsal telson spines normal

6

6. Fourth thoracic sternite with transverse ridge with keyhole shaped median notch; R.1+8$9 / 2$ $P$. terangeri Bruce

Fourth thoracic sternite lacking transverse ridge with median notch .. 7

7. Major chela more slender, 5.2 times longer than wide, fingers 0.4 of palm length; ambulatory dactyl with or without minute accessory tooth, ca 0.2 of unguis length; R.9/2.

$P$. nomadophophila Berggren

Major chela more robust, 4.1 times longer than wide; fingers 0.3 of palm length; ambulatory dactyl with distinct accessory tooth, ca 0.7 of unguis length; R.10/2 ..... P. delagoae Barnard

8. First pereiopod with carpus much shorter than chela, subequal to palm; R. 6/1

$P$. batei Holthuis

First pereiopod with carpus subequal to or longer than chela

9. Second pereiopods slender, subequal, with fingers of major chela subequal to palm, carpus more than half palm length; R. 9-10/2

$P$. sinensis Bruce

Second pereiopods robust, markedly unequal, with fingers of major chela distinctly shorter than palm, major carpus ca half palm length; R. $8-11 / 1-2$. $P$. burrup sp. nov. 


\section{Periclimenes holthuisi Bruce, 1969}

Periclimene's holthuisi Bruce, 1969: 258. - Bruce, 1982a: 244, fig. 7. - Li, 2000: 190, fig. 241. Davie, 2002: 327. - Okuno, 2005: 273, fig. 6CD.

\section{Material examined}

Western Australia, Dampier Archipelago. WAM C 28061 (1 ovig. female), stn DA3/99/41, Georgeff Reef $\left(20^{\circ} 29.339^{\prime} \mathrm{S}, 116^{\circ} 36.798^{\prime} \mathrm{E}\right), 1.0-4.0 \mathrm{~m}, 28-$ 29.08.1999.

\section{Host}

Actinodendron sp. [Actinaria]. Not previously reported in association with this anemone genus.

\section{Remarks}

The single specimen, CL $3.25 \mathrm{~mm}$, corresponds exactly with the original description and has a rostral dentition of $1+9 / 1$, with an extra minute vestigial tooth distally. The ophthalmic somite bears a short bec ocellaire. The second pereipod chelae are precisely as illustrated by Okuno (2004, fig. 6 CD) with a well marked proximal gape, the cutting edges are densely provided with numerous groups of long simple setae, distinctly more than shown in the topotypic material illustrated by Bruce (1982a, fig. 7C). The third ambulatory pereiopod has the propod with a pair of long distoventral spines and two pairs and a single spine on the distal half of the ventral margin. The colouration is unrecorded.

This represents a new record for Western Australia.

\section{Australian Distribution}

Reported from Shark Bay by Bergren (1997b). Northern Territory: Sandy I. No. 2, Cobourg Pen. (Bruce, 1983); East Pt, Darwin (Bruce, 1988a); Port Essington; Port Bremer, Orontes Reef, Cobourg Pen. (Bruce and Coombes, 1995); South Shell I., Darwin Harbour (Bruce and Coombes, 1997). Queensland: Bowen (Bruce, 1977b); Peloris I. (Bruce, 1977a); Morton Bay (Wadley, 1978; Young and Wadley, 1979; Davie et al., 1998); Heron I., Capricorn Is (Bruce, 1981; Coleman, 1988)

\section{Further Distribution}

Type locality: Lung Ha Wan, Hong Kong. Recorded from the Red Sea, Jordan, Zanzibar, Maldive Islands, Sri Lanka, Malaya, Singapore, Indonesia, Vietnam, China, Hong Kong, South China Sea, Japan, Philippines, Papua New Guinea, New Caledonia, Caroline Islands, and Marshall Islands.

\section{Periclimenes incertus Borradaile, 1915}

Periclimenes (Cristiger) incertus Borradaile, 1915 : 210. - Borradaile, 1917: 364, pl. 53 fig. 7
Periclimenes (Periclimenes) impar Kemp, 1922: 147, figs 16-17, pl. 3 fig. 1.

Periclimenes (Periclimenes) incertus. - Holthuis, 1959: 193

Periclimenes incertus, - Davie, 2002: 327.

\section{Material examined}

Western Australia, Dampier Archipelago. (1) WAM C 25431 (1 male), stn DA2/99/31, off Courtney Head, Malus I. (20²9.49'S, $116^{\circ} 40.61^{\prime} \mathrm{E}$ to $\left.20^{\circ} 29.66^{\prime} \mathrm{S}, 116^{\circ} 41.01^{\prime} \mathrm{E}\right)$, rake box dredge, 11.5 m, 18.07.1999; (2) WAM C 28096 (1 female), stn DA3/99/68, off Bluff Pt, Enderby I. $\left(20^{\circ} 40.93^{\prime}\right.$ S, $116^{\circ} 33.21^{\prime} \mathrm{E}$ to $\left.20^{\circ} 40.63^{\prime} \mathrm{S}, 116^{\circ} 33.36^{\prime} \mathrm{E}\right)$, rake box dredge, 9.0-9.2 m, 23.07.1999; (3) WAM C 27706 (1 ovig. female), stn DA2/99/75, off Goodwyn I. $\left(20^{\circ} 32.16^{\prime} \mathrm{S}, \quad 116^{\circ} 33.70^{\prime} \mathrm{E}\right.$ to $20^{\circ} 31.70^{\prime} \mathrm{S}$, $\left.116^{\circ} 33.20^{\prime} \mathrm{E}\right)$, rake box dredge, $19.0-14.0 \mathrm{~m}$, 25.07.1999; (4) WAM C 28091 (1 ovig. female), stn DA3/99/65, Enderby I. (20³8.31'S, $116^{\circ} 38.46^{\prime} \mathrm{E}$ to $\left.20^{\circ} 38.77^{\prime} \mathrm{S}, 116^{\circ} 38.54^{\prime} \mathrm{E}\right), 13.0-15.0$ m, 06.09.1999, (5) WAM C 26625 (1 spm), stn DA2/99/56, off Roly Rocks (20³0.10'S, $116^{\circ} 28.27^{\prime} \mathrm{E}$ to $20^{\circ} 29.88^{\prime} \mathrm{S}, 116^{\circ} 27.93^{\prime} \mathrm{E}$ ), rake box dredge, 33.0-34.5 m, 21.07.1999; (6) WAM C 276095 (3 ovig. females), stn DA3/99/55, off Roly Rocks $\left(20^{\circ} 28.45^{\prime} \mathrm{S}, 116^{\circ} 27.43^{\prime} \mathrm{E}\right.$ to $20^{\circ} 27.98^{\prime} \mathrm{E}$, $\left.116^{\circ} 27.54^{\prime} \mathrm{E}\right)$, rake box dredge, $37.5-38.0 \mathrm{~m}$, 21.07.1999.

\section{Remarks}

The specimens have a rostral dentition of $1+6-7 / 1$.

\section{Hosts}

Specimens (4) and (6) were associated with sponges.

\section{Australian Distribution}

Western Australia: North West C. (Balss, 1921 as Palaemonella biunguiculata); Shark Bay (Berggren, 1997b); central Kimberleys, Churchill reef, Jesseux I. (Berggren, 1997c). Northern Territory: East Pt, Darwin (Bruce, 1988a); Sandy I. No. 2; Port Essington; Orontes Reef; Barrow Bay, Cobourg Pen. (Bruce and Coombes, 1995); Channel Rock; Weed Reef; South Shell I., Darwin Harbour (Bruce and Coombes, 1997). Queensland: Heron I., Capricorn Is (Bruce, 1981).

\section{Further Distribution}

Type locality: South Nilandu Atoll, Maldive Islands. Recorded from the Yemen, Kenya, Zanzibar, Tanganyika, Madagascar, Maldive Islands, Sri Lanka, Andaman Islands, Singapore, Indonesia, Philippines, Papua New Guinea and New Caledonia. 
Periclimenes magnificus Bruce, 1979

Periclimenes magnificus Bruce, 1979: 195, figs 1-5, pl. 1A-C. - Li, 2000: 212, fig. 274. - Davie, 2002: 330.

\section{Material examined}

Western Australia, Dampier Archipelago. WAM C 28097 ( 2 juvs), stn DA3/99/70, Nelson Rocks (20²7.441'S, $\left.116^{\circ} 39.588^{\prime} \mathrm{E}\right), 5.0-7.0 \mathrm{~m}, 08.09 .1999$.

\section{Remarks}

This is a new record for the Western Australian fauna. The specimen has a rostral dentition of $1+7 / 1$.

\section{Australian Distribution}

Northern Territory: Cobourg Peninsula (Bruce and Coombes, 1995). Queensland: Low Isles (?) (Stephenson et al., 1931); Heron Island, Wistari Reef (Bruce, 1979c; 1980; 1981; 1986).

\section{Further Distribution}

Type locality: Wistari Reef, Capricorn Islands, Queensland. Known from Thailand, Indonesia, Japan, Philippine Islands, Papua New Guinea and New Caledonia.

\section{Periclimenes novaffinis Bruce and Coombes, 1997}

Periclimenes novaffinis Bruce and Coombes, 1997: 101. - Davie, 2002: 330

\section{Material examined}

Western Australia, Dampier Archipelago. WAM C 28064 (1 male, 1 ovig. female), stn DA3/99/41, Georgeff Reefs $\left(20^{\circ} 29.339^{\prime} \mathrm{S}, 116^{\circ} 36.798^{\prime} \mathrm{E}\right), 1.0-4.0$ $\mathrm{m}$, coll. P. Morrison, 28-29.09.1999.

\section{Host}

Zygometra microdiscus (Bell, 1882), Z 5361, a new host record [Echinodermata, Crinoidea].

\section{Remarks}

The specimens have a rostral dentition of 6/1, 9/1. The male has a slightly more slender rostrum than the female.

The original material was found in association with the crinoids Zygometra andromeda, $Z$. punctata and $Z$. elegans

\section{Australian Distribution}

New to the Western Australian fauna. Northern Territory: Type locality: East Point, Darwin Harbour (Bruce and Coombes, 1997). Also known from Fannie Bay and Bullocky Point, Darwin (Bruce and Coombes, 1997).

\section{Further Distribution}

Not known outside Australian waters.

\section{Periclimenes soror Nobili, 1904}

Periclimenes soror Nobili, 1904: 232. - Bruce, 1978c: 299, figs 1-6. - Li: 237, fig. 316. - Davie, 2002: 332.

Periclimenes (Periclimenes) soror. - Holthuis, 1952: 51, fig. 17.

\section{Material examined}

(1) WAM C 25702 (1 ovig. female), stn DA1/98/04, Legendre I. (20 $\left.24.320^{\prime} \mathrm{S}, 116^{\circ} 56.108^{\prime} \mathrm{E}\right), 12.0-18.0 \mathrm{~m}$, 18.10.1998; (2) WAM C 25699 (1 male), stn DA1/98/ 04 , Legendre I. $\left(20^{\circ} 24.320^{\prime} \mathrm{S}, 116^{\circ} 56.108^{\prime} \mathrm{E}\right), 12.0-18.0$ m, 18.10.1998.

\section{Remarks}

The female specimen lacks both second pereiopod and has a rostral dentition of $14 / 0$. The male has the dentition of $11 / 0$.

\section{Host}

The male was associated with Culcita novaeguineae Müller and Tröschel [Asteroidea].

\section{Australian Distribution}

Western Australia: Kendrew I., Dampier Arch.; Exmouth (Bruce, 1978h); Cartier and Hibernia Reefs (Bruce, 1992c); Shark Bay (Berggren, 1997b). Northern Territory: Darwin, Dudley Pt (Bruce, 1983a); New Year I., Cobourg Pen., 16 m (Bruce and Coombes, 1995). Queensland: Green I., Fairfax I. (Bruce, 1971); Bowen Reef, Lodestone Reef (Bruce, 1977a); Lodestone Reef, 3 m (Zann, 1980); Chapman I.; Beaver Reef (Bruce, 1978h); Heron I., Wistari Reef; Lizard I. (Bruce, 1983a); Moreton Bay (Davie et al., 1998). New South Wales: Cuwatong (Bruce, 1978c); Jervis Bay, $15 \mathrm{~m}$ (Ellis, 1987); Ulladulla, $30 \mathrm{~m}$, on "firebrick sea-star" (Sullivan, 1997).

\section{Further Distribution}

Type locality: Jibuti. Reported from Saudi Arabia, Kenya, Zanzibar, Tanganyika, Madagascar, Seychelle Islands, Chagos Islands, Sri Lanka, Malaya, Indonesia, Vietnam, China, Hong Kong, Taiwan, Japan, Philippines, Sabah, Bismarck Archipelago, Solomon Islands, Papua New Guinea, New Caledonia, Marshall Islands, Mariannas Islands, Fijian Islands, Hawaiian Islands, Society Islands, Tuamotu Islands. Also western Mexico, Panama and Colombia (Bruce, 1978c).

\section{Philarius gerlachei (Nobili, 1905)}

Harpilius gerlachei Nobili, 1905: 160. - Nobili, 1906: 45, pl. 4 fig. 10. - Kemp, 1922: 238, figs 74-75.

Philarius gerlachei. - Holthuis, 1952: 152, fig. 69. Li, 2000: 251, fig. 334. - Davie, 2002: 334. 
Material examined

See below.

\section{Remarks}

The pair of second pereiopod chelae found in association with Coralliocaris sp. (WAM C 28050) correspond exactly with the figure of that appendage provided by Kemp (1922, fig. 75, as Harpilius gerlachei). The lack of a distoventral tooth on the merus distinguishes them from $P$. imperialis (Kubo). Both Philarius species are common associates of Acropora coral hosts, as are most Coralliocaris species.

\section{Australian Distribution}

Western Australia: Hibernia Reef (Bruce, 1992); central Kimberleys, East Montlivet. (Berggren, 1997c). Northern Territory: Cobourg Peninsula (Bruce and Coombes, 1995). Queensland: Willis Island; Bet Reef; Restoration Rock; Heron Island and Wistari Reef, Capricorn Islands (Patton, 1966); One Tree Island; Diamond Islet (Bruce, 1977b); Heron Island (Bruce, 1981).

\section{Further Distribution}

Type locality: Arzana Island, United Arab Emirates. Reported from Egypt, Saudi Arabia, Oman, Sudan, Kenya, Zanzibar, Tanganyika, Moçambique Channel, Comoro Islands, Seychelle Islands, Réunion, India, Indonesia, Vietnam, China, Japan, Papua New Guinea, Solomon Islands, Marshall Islands, Samoan Islands, Fijian Islands, Kiribati and Tuamotu Islands.

\section{Philarius imperialis (Kubo, 1940)}

Harpilius imperialis Kubo, 1940: 1, figs 1-3.

Philarius imperialis. - Holthuis, 1952: 15. - Li, 2000: 252, fig. 335. - Davie, 2002: 334.

\section{Material examined}

Western Australia, Dampier Archipelago. (1) WAM C 29215 (1 female), stn DA3/99/36, Malus I. (2030.050'S, 116 40.594'E), 6.0-14.0 m, 27.08. 1999; (2) WAM C 25303 (1 male), stn DA1/98/03, Legendre I. $\left(20^{\circ} 24.320^{\prime} \mathrm{S}, 116^{\circ} 56.108^{\prime} \mathrm{E}\right), 2.0-15.0 \mathrm{~m}$, 18.10.98.

\section{Host}

Acropora spp [Scleractinia, Acroporidae].

\section{Remarks}

This is a new addition to the Western Australian fauna. The female specimen had a rostral dentition of $8 / 1$ and the male, $6 / 1$.

\section{Australian Distribution}

Reported from central Kimberleys, Albert Reef
(Berggren, 1997c). Northern Territory: Port Essington, Coral Bay (Bruce, 1983b); Cobourg Peninsula (Bruce and Coombes, 1995). Queensland: Restoration Rock; Heron Island, Capricorn Islands (Patton, 1966); Queensland: Heron Island (Bruce, 1981).

\section{Further Distribution}

Type locality: Nankin-hama, Haha-jima, Ogasawa Islands. Reported from Saudi Arabia, Israel, Kenya, Zanzibar, Tanganyika, Seychelle Islands, Réunion, Singapore, Vietnam, China, Japan, Papua New Guinea, Caroline Islands, Marshall Islands.

\section{The Pontoniine Fauna of Western Australia}

Original and major descriptions, and name changes only are given short citations only. Full citations are provided in $\mathrm{Li}$ (2000) and Davie (2002)). Berggren (1997) lists two unidentified species of Periclimeneus and seven species of Periclimenes which are not included in the following list and may overlap with some taxa in the above report.

\section{Anchistus custos (Forsskål, 1775)}

Cancer custos Forskäl, 1775, Descrpt. Anim., xxi, 94.

Harpilius inermis Miers, 1884, Rep. Zool. Coll. Alert, 291, pl. 32, fig. B.

Anchistus inermis. - Borradaile, 1898, Ann. Mag. Nat. Hist. (7) 2: 387.

Anchistus custos. - Holthuis, 1952, Siboga Exped. Mon. 39a ${ }^{10}$ : 105-109, figs 43-44.

Western Australian Distribution: Shark Bay (as Harpilius inermis) (Miers, 1884; Berggren, 1997b); Monte Bello Is (Rathbun, 1914, as A. inermis); Denham (Jones, 1990); Sunday I., Irvine I., southern Kimberleys (Davie and Short, 1995); central Kimberleys, East Berthier I. (Berggren, 1997c).

\section{Anchistus miersi (De Man, 1888)}

Harpilius Miersi De Man, 1888, J. Linn. Soc. Lond., Zool. 22: 274, pl. 17, figs 6-10.

Anchistus miersi. - Borradaile, 1898, Ann. Mag. Nat. Hist. (7) 2:387.

Marygrande mirabilis Pesta, 1911, Zool. Anz. 38: 572.

Western Australian Distribution: Hibernia Reef (Bruce, 1992); central Kimberleys, White, Hedley, Lamarck and Prud Hoe Is (Berggren, 1997c); Shark Bay (Berggren, 1997b). 
Apopontonia orbitospinata (Bruce, 1988)

Periclimeneaus orbitospinatus Bruce, 1969, Zool. Meded., Leiden 44: 160-16.

Apopontonia tridentata Bruce, 1988, J. Nat. Hist. 22: 1270-1276, figs 4-7.

Apopontonia orbitospinata. - Bruce, 2001, Zool. Meded., Leiden 152-157, fig. 4.

Western Australian Distribution: Northwest Shelf, $54 \mathrm{~m}$ (Bruce, 1988c); Arafura Sea, $60 \mathrm{~m}$ (Debelius, 1999; as A. tridentata).

Araiopontonia odontorhyncha Fujino and Miyake, 1970

Araiopontonia odontorhyncha Fujino and Miyake, 1970, Ohmu 3 (1): 2-10, figs. 1-4.

Western Australian Distribution: Cartier Reef (Bruce, 1992).

\section{Carinopontonia paucipes Bruce, 1988}

Carinopontonia paucipes Bruce, 1988, J. Nat. Hist., 22: 1264-1274, figs 1-3.

Western Australian Distribution: Northwest Shelf, $19^{\circ} 04.4^{\prime} \mathrm{S}, 118^{\circ} 47.55^{\prime} \mathrm{E}, 83 \mathrm{~m}$ (Bruce, 1988b).

\section{Conchodytes biunguiculatus (Paul'son, 1875)}

Pontonia biunguiculatus Paul'son, 1875, Crust. Red Sea: 111-112, pl. 15 fig. 1.

Western Australian Distribution: Hibernia Reef (as C. kempi, Bruce, 1992).

\section{Conchodytes maculatus Bruce, 1989}

Conchodytes maculates Bruce, 1989, Crustaceana 56 (2): 182-191, figs 1-6.

Western Australian Distribution: Off Cape Leveque (Bruce, 1989b); Shark Bay (Berggren, 1997b).

Conchodytes meleagrinae Peters, 1852. See above.

Conchodytes monodactylus Holthuis, 1952. See above.

\section{Conchodytes tridacnae Peters, 1852}

Conchodytes tridacnae Peters, 1852, Ber. Verh. Akad. Wiss. Berlin 1852: 594.

Western Australian Distribution: Hibernia Reef (Bruce, 1992).

\section{Coralliocaris graminea (Dana, 1852)}

Oedipus gramineus Dana, 1852, Proc. Acad. Nat. Sci. Philad. 6: 25.

Coralliocaris graminea. - Stimpson, 1860, Proc. Acad. nat. Sci. Philad. 1860: 38.

Western Australian Distribution: Shark Bay (Jones, 1990; Berggren, 1997b); Rottnest I. (Jones and Morgan, 1993); central Kimberleys, East Montlivet and Prud Hoe Is (Berggren, 1997c).

Coralliocaris venusta Kemp, 1922. See above.

Coralliocaris viridis Bruce, 1974. See above.

Dasella ansoni Bruce, 1983

Dasella ansoni Bruce, 1983, Beagle, Occ. Pap. N.T. Mus. 1 (3): 22-28, figs 1-5.

Western Australian Distribution: Shark Bay, in Herdmania momus (Berggren, 1999).

Dasycaris zanzibarica Bruce, 1973

Dasycaris zanzibarica Bruce, 1973, Crustaceana 24(3): 247-257, figs 1-6.

Western Australian Distribution: central Kimberleys, Jesseux I. (Berggren, 1997c).

Exoclimenella maldivensis Duris and Bruce, 1995

Exoclimenella maldivensis Duris and Bruce, 1995, J. Nat. Hist. 29: 622-631, figs 1-5.

Western Australian Distribution: Cartier Reef, 12-18m (Duris and Bruce, 1995).

\section{Exopontonia malleatrix Bruce, 1988}

Exopontonia malleatrix Bruce, 1988, J. Crust. Biol. 81 (1): 123-130, figs 1-5.

Western Australian Distribution: Ashmore Reef (Bruce, 1988c).

Hamodactylus aqabai Bruce and Svoboda, 1983

Hamodactylus aqabai Bruce and Svoboda, 1983, Zool. Verhand., Leiden 205: 26-35, fig 10.

Western Australian Distribution: Cartier Reef (Bruce, 1992).

\section{Hamodactylus boschmai Holthuis, 1952}

Hamodactylus boschmai Holthius, 1952, Siboga Exped. Mon. 39 $\mathbf{a}^{10}$ : 209-212, figs 102-104.

Western Australian Distribution: central Kimberleys, De Freycinet I. (Berggren, 1997c).

Hamodactylus noumeae Bruce, 1970

Hamodactylus noumeae Bruce, 1970, J. Zool. Lond. 160: 539-541, fig. 2.

Western Australian Distribution: Cartier and Hibernia Reefs (Bruce, 1992); central Kimberleys, Alberet, Churchill reefs, Cassini I., East Montlivet I. (Berggren, 1997c).

\section{Hamodactylus sp. Tsareva, 1980: 125.}

The identity of this species is uncertain and attempts to locate the specimens have been unsuccessful. Twelve specimens were collected 
from $1.5 \mathrm{~m}$ and $12 \mathrm{~m}$, from Scott Reef, from Acropora colonies, and were in poor condition making identification uncertain (Duris, pers. comm.). No Hamodactylus species have so far been found in association with scleractinian hosts.

\section{Hamopontonia corallicola Bruce, 1970}

Hamopontonia corallicola Bruce, 1970 , Crustaceana 18 (1): 41-48, figs 1-4.

Western Australian Distribution: Sunday I.; Montgomery Reef, southern Kimberleys (Davie and Short, 1995, as Hamodactylus corallicola); Long I., Vansittart Bay, eastern Kimberleys (Davie and Short, 1996); central Kimberleys, Churchill Reef (Berggren, 1997c).

\section{Hamopontonia aff. corallicola}

Hamopontonia aff. corallicola. - Berggren, $1997 \mathrm{c}$ (unpub. report, p. 89).

Western Australian Distribution: Shark Bay (Berggren, 1997b); central Kimberleys, Slate Is (Berggren, 1997c).

Harpiliopsis beaupresii (Audouin, 1825). See above.

Harpilius bayeri (Holthuis, 1981). See above.

\section{Harpilius consobrinus De Man, 1902}

Harpilius consobrinus De Man, 1902, Abh. Senckenb. naturf. Ges. 25: 836-840, pl. 26, fig. 54. Periclimenes consobrinus. - Bruce, 1972, Proc. Symp. Corals and Coral Reefs; Bruce, 1969. Mar. Biol. Soc. India: 403, 409, 412 (key), fig. 1b.

Harpilius consobrinus, - Bruce, 2004, Zootaxa 293: 6 .

Western Australian Distribution: Hibernia Reef (Bruce, 1992).

\section{Ischnopontonia lophos (Barnard, 1962)}

Philarius lophos Barnard, 1962, Crustaceana 3 (3): 242-243, fig. 2.

Ischnopontonia lophos. - Bruce, 1966, Bull. Mar. Sci. Univ. Miami 16 (3): 584

Western Australian Distribution: central Kimberleys, Rob Roy Reef, Jamieson Reef, Maret Is, East Montlivet I. (Berggren, 1997c).

Kemponia amymone (De Man, 1902). See above.

\section{Kemponia anacanthus (Bruce, 1989)}

Periclimenes anacanthus Bruce, 1989, Beagle, Rec. N.T. Mus. Arts and Sci. 58: 105-114, figs 15.

Kemponia anacanthus. - Bruce, 2004, Zootaxa 293: 12.
Western Australian Distribution: Shark Bay (Berggren, 1997b); Cape Londonderry, eastern Kimberleys (Davie and Short, 1996).

Kemponia andamanensis (Kemp, 1922). See above.

Kemponia elegans (Paul'son, 1875). See above.

Kemponia grandis (Stimpson, 1860). See above.

Kemponia aff. suvadivensis (Borradaile, 1915)

Periclimenes (Falciger) suvadivensis Borradaile, 1915, Ann. Mag. nat. Hist. (8) 15: 212.

Kemponia suvadivensis. - Bruce, 2004, Zootaxa 293: 19.

Western Australian Distribution: Sunday I., Whirlpool Pass, southern Kimberleys (Davie and Short, 1995).

\section{Kemponia tenuipes (Borradaile, 1898)}

Periclimenes tenuipes Borradaile, 1898, Ann Mag. nat. Hist. (7) 2: 384

Kemponia tenuipes. - Bruce, 2004, Zootaxa 293: 19-20.

Western Australian Distribution: Hibernia Reef (Bruce, 1992c).

\section{Manipontonia psamathe (De Man, 1902)}

Urocaris psamathe De Man, 1902, Abh. Senckenb. naturf. Ges. 25: 816-822, pl. 25 fig. 51. Periclimenes (Ancylocaris) psamathe - Kemp, 1922, Rec. Indian Mus. 24: 173.

Periclimenes (Harpilius) psamathe. - Holthuis, 1952, Siboga Exped. Mon. 39a ${ }^{10}:$ 61, fig. 23.

Manipontonia psamathe. - Bruce, Okuno and Li, 2005, Zootaxa, 926: 6-8, figs 1-3.

Western Australian Distribution: Shark Bay (Berggren, 1997b); central Kimberleys, Rob Roy Reef, Gibbings Reef, Jesseux I., Cassini I., Macleay I. (Berggren, 1997c).

\section{Neopontonides sp. Tsareva, 1980: 125.}

The identity of this species is uncertain and attempts to locate the specimens have been unsuccessful. Neopontonides is an AtlanticCaribbean genus not known from the Indo-WestPacific region. Tsareva's specimens were reported from $7.0 \mathrm{~m}$ in association with Seriatopora, the shrimp associates of which are comparatively well studied.

\section{Notopontonia platycheles Bruce, 1991}

Notopontonia platycheles Bruce, 1999, J. Crust. Biol. 11 (4): 607-628, figs 1-14.

Western Australian Distribution: Fitzgerald 
Reserve (Berggren, 1997a); Shark Bay (Berggren, 1997b).

\section{Palaemonella crosnieri Bruce, 1978}

Palaemonella crosnieri Bruce, 1978, Zool. Journ. Linn. Soc. 62: 210-214, figs 2-4.

Western Australian Distribution: Cartier Reef (Bruce, 1992).

\section{Palaemonella foresti Bruce, 2002}

Palaemonella foresti Bruce, 2002, Crustaceana 75 (3-4): 277-298, figs 1-4.

Western Australian Distribution: Cockburn Sound (Bruce, 2002b).

Palaemonella pottsi Borradaile, 1915. See above.

Palaemonella rotumana (Borradaile, 1898). See above.

\section{Palaemonella tenuipes Dana, 1852}

Palaemonella tenuipes Dana, 1852, Proc. Acad. nat. Sci., Philad. 6: 25.

Western Australian Distribution: C. Jaubert (Balss, 1921).

\section{Parapontonia nudirostris Bruce, 1968}

Parapontonia nudirostris Bruce, 1968, Bull. Mus. Nat. Hist. nat., Paris (2)39(6). - Bruce 1967: 11491157, figs 1-5.

Western Australian Distribution: Shark Bay (Berggren, 1997b).

\section{Periclimenaeus arabicus Calman, 1939}

Periclimenes (Periclimenaeus) arabicus Calman, 1939, Sci. Rep. John Murray Exped. 6: 210-211, fig. 4.

Western Australian Distribution: Shark Bay (Berggren, 1997b).

\section{Periclimenaeus bidentatus Bruce, 1970}

Periclimenaeus bidentatus Bruce, 1970, Zool. Meded., Leiden 44 (21): 305-307.

Western Australian Distribution: Hibernia Reef (Bruce, 1992); Shark Bay (Berggren, 1997b).

Periclimenaeus hecate (Nobili, 1904) See above.

Periclimenaeus kottae Bruce, 2005

Periclimenaeus kottiae Bruce, 2005, Rec. $W$. Aust. Mus. 22: 325-331, figs 1-3.

Western Australian Distribution: Ashmore Reef (Bruce, 2005).

\section{Periclimenaeus matherae Bruce, 2005}

Periclimenaeus matherae Bruce, 2005, Rec. $W$. Aust. Mus. 22: 331-338, figs 4-8.
Western Australian Distribution: Ashmore Reef (Bruce, 2005).

Periclimenaeus minutus Holthuis, 1952

Periclimenaeus minutus Holthuis, 1952, Siboga Exped. Mon. 39a ${ }^{10}:$ 134-137, figs 57-59.

Western Australian Distribution: central Kimberleys, Churchill Reef, Albert Reef (Berggren, 1997c).

Periclimenaeus pachydentatus Bruce, 1969

Periclimenaeus pachydentatus Bruce, 1969, Zool. Meded., Leiden 44 (12): 162-163.

Western Australian Distribution: Hibernia Reef (Bruce, 1992; Berggren, 1997b).

\section{Periclimenaeus rastrifer Bruce, 1980}

Periclimenaeus rastrifer Bruce, 1980, Cahiers Indo-Pacifique 2(1): 27-33, figs 12, $13 \mathrm{~A}, \mathrm{~B}$.

Western Australian Distribution: Shark Bay (Berggren, 1997b).

\section{Periclimenaeus stylirostris Bruce, 1969}

Periclimenaeus stylirostris Bruce, 1969, Zool. Meded., Leiden 44(12): 167-168. - Bruce, 1972, Pacific Sci. 26 (1): 68-75, figs 2-6.

Western Australian Distribution: Shark Bay (Berggren, 1997b).

Periclimenella spinifera (De Man, 1902). See above.

\section{Periclimenes aesopius (Bate 1863).}

Anchistia aesopia Bate, 1863, Proc. Zool. Soc. Lond. 1863: 502-503, pl. 41, fig. 5.

Periclimenes aesopius. - Kemp, 1922, Rec. Indian Mus. 24: 142-143, fig. 12.

Periclimenes aesopius. - Bruce, 1977, Aust. Zool. 19 (2): 217-226, figs 1-29, 34.

Western Australian Distribution: Princess Royal Harbour (Kirkman et al., 1991); Fitzgerald Reserve (Berggren, 1997a).

Periclimenes affinis (Zehntner, 1894). See above.

Periclimenes alegrias Bruce, 1986. See above.

\section{Periclimenes amboinensis (De Man, 1888)}

Anchistia amboynensis De Man, 1888, Arch. Naturgesch. 53 (1): 546-548, pl. 22a, fig. 2.

Periclimenes amboinensis. - Borradaile, 1898, Ann. Mag. nat. Hist. (7) 2: 383.

Western Australian Distribution: Cartier Reef (Bruce, 1992). 


\section{Periclimenes batei Holthuis, 1959}

Palaemonella orientialis Bate, 1888, Rep. Voy Challenger Exped., Zool, 24: 278.

Palaemonella batei Borradaile, 1917, Trans. Linn Soc. Lond., Zool. (2) 17: 357, 358.

Periclimenes (Periclimenes) batei. - Holthuis, 1959, Zool. Meded., Leiden 36 (11): 195-197.

Western Australian Distribution: Hibernia Reef (?) (Bruce, 1992).

\section{Periclimenes brevicarpalis (Schenkel, 1902)}

Ancylocaris brevicarpalis Schenkel, 1902, Verh. naturf. Ges. Basel 13: 563, pl. 13, fig. 21.

Periclimenes hermitensis Rathbun, 1914, Proc. zool. Soc. Lond. 1914: 655, pl. 1 figs 1-3.

Periclimenes (Ancylocaris) brevicarpalis. Kemp, 1922, Rec. Indian Mus. 24: 185-191, figs 40-42, pl. 67.

Periclimenes (Harpilius) brevicarpalis. Holthuis, 1952, Siboga Exped. Mon. 39a ${ }^{10}: 69-73$, fig. 27.

Western Australian Distribution: Monte Bello Is, Hermit I. (Rathbun, 1914, as P. hermitensis); Hibernia Reef (Bruce, 1992); Shark Bay (Berggren, 1997b); Central Kimberleys, Slate Is (Berggren, 1997c).

Periclimenes burrup sp. nov. See above.

Periclimenes aff. cobourgi. - Bruce, 1995

Western Australian Distribution: Shark Bay (Berggren, 1997b).

\section{Periclimenes commensalis Borradaile, 1915}

Periclimenes (Cristiger) commensalis Borradaile, 1915, Ann. Mag. Nat. Hist. (8)15: 211. Borradaile, 1917, Trans. Linn. Soc., Lond., Zool. (2)17: 364 .

Western Australian Distribution: Shark Bay (Berggren, 1997b); central Kimberleys, Jameson Reef, Cassini I. (Berggren, 1997c).

Periclimenes aff. cristimanus Berggren, 1997c

Periclimenes aff. cristimanus. - Berggren, 1997c, Mar. Biol. Surv. Central Kimb. Coast, WA, Univ. WA: 89

Western Australian Distribution: central Kimberleys, White I., East Montlivet I. (Berggren, 1997c).

Periclimenes aff. grandis. See above.

Periclimenes holthuisi Bruce, 1969. See above.

Periclimenes hongkongensis Bruce, 1969

Periclimenes hongkongensis Bruce, 1969, Zool Meded., Leiden, 43 (20): 259-260. - Bruce, 1982,
Proc. First Internat. Mar. Biol. Wksp., Hong Kong, 1980: 247-252, figs 8-10.

Western Australian Distribution: central Kimberleys, Macleay I. (Berggren, 1997c).

Periclimenes imperator Bruce, 1967

Periclimenes imperator Bruce, 1967, Zool Verhand, Leiden 87: 53-62, figs 23-25.

Western Australian Distribution: Cartier Reef (Bruce, 1992).

\section{Periclimenes indicus (Kemp, 1915)}

Urocaris indica Kemp, 1915, Mem. Indian Mus 5: 275-279, fig. 26, pl. 13, fig. 9.

Periclimenes (Periclimenes) indicus. - Kemp, 1922, Rec. Indian. Mus. 24: 144, fig. 13.

Western Australian Distribution: C. Londonderry, eastern Kimberleys (Davie and Short, 1996).

Periclimenes incertus Borradaile, 1915. See above.

Periclimenes inornatus Kemp, 1922

Periclimenes (Ancylocaris) inornatus Kemp, 1922, Rec. Indian Mus. 24: 191-194, figs 44-46.

Western Australian Distribution: Hibernia Reef (Bruce, 1992).

\section{Periclimenes kempi Bruce, 1969}

Periclimenes kempi Bruce, 1969, Zool. Meded., Leiden 43 (20): 260-261.

Western Australian Distribution: Cartier Reef (Bruce, 1992); Shark Bay (Berggren, 1997b) central Kimberleys, Slate Is (Berggren, 1997c).

\section{Periclimenes mahei Bruce 1969}

Periclimenes mahei Bruce, 1969, Zool. Meded., Leiden, 43 (20): 263-264.

Western Australian Distribution: Pt Quobba (Black and Prince, 1983; Bruce, 1990a).

Periclimenes magnificus Bruce, 1979. See above.

Periclimenes novaffinis Bruce and Coombes, 1997. See above.

\section{Periclimenes aff. obscurus Kemp, 1922}

Periclimenes (Periclimenes) obscurus Kemp, 1922, Rec. Indian Mus. 24: 144-146, figs 14-15.

Western Australian Distribution: Fitzgerald Reserve (Berggren, 1997a); central Kimberleys Churchill Reef, Jameson Reef (Berggren, 1997c).

Periclimenes seychellensis Borradaile, 1915 Periclimenes (Falciger) seychellensis Borradaile, 1915, Ann. Mag. nat. Hist. (8) 15: 212 . - 
Borradaile, 1917, Trans. Linn. Soc. Lond, Zool. (2) 17: 324, 375, pls. 54-55, fig. 14.

Western Australian Distribution: central Kimberleys, Colbert Is (Berggren, 1997c).

Periclimenes sinensis Bruce, 1969. See above.

Periclimenes soror Nobili, 1904. See above.

\section{Periclimenes venustus Bruce, 1990}

Periclimenes venustus Bruce, 1990, Indo-Malay. Zool. 6: 230-240, figs 1-6, 7a, 8a.

Western Australian Distribution: Scott Reef (Bruce, 1990c); Abrolhos Is (Steene, 1990); Hibernia Reef (Bruce, 1992); Irvine I., southern Kimberleys (Davie and Short, 1995); central Kimberleys, Churchill Reef, East Berthier I. (Berggren, 1997c).

\section{Periclimenes zanzibaricus Bruce, 1967}

Periclimenes zanzibaricus Bruce, 1967, Zool. Verhand., Leiden 87: 62-72, figs 26-29.

Western Australian Distribution: Geraldton (Bruce, 1973).

\section{Periclimenoides odontodactylus (Fujino and Miyake, 1968)}

Periclimenaeus odontodactylus Fujino and Miyake, 1968, Ohmu 1(3): 85-90, figs. 1-2. Periclimenoides odontodactylus. - Bruce, 1990, Proc. $2^{\text {nd }}$ Internat: Mar. Biol. Wksp., Hong Kong, 1986 2: 617-618, figs 2-3.

Western Australian Distribution: Northwest Shelf (Bruce, 1990).

Philarius gerlachei (Nobili, 1905). See above.

Philarius imperialis (Kubo, 1940). See above.

\section{Platycaris latirostris Holthuis, 1952}

Platycaris latirostris Holthuis, 1952, Siboga Exped. Mon. 39a ${ }^{10}:$ 173-176, figs 85-86.

Western Australian Distribution: central Kimberleys, Rob Roy Reef, Jameson Reef (Berggren, 1997c).

\section{Platypontonia hyotis Hipeau-Jacquotte, 1971}

Platypontonia hyotis Hipeau-Jacquotte, 1971, Crustaceana 20(2): 126-139, figs 1-7.

Western Australian Distribution: Shark Bay (Berggren, 1997b).

\section{Pontoniopsis comanthi Borradaile, 1915.}

Pontoniopsis comanthi Borradaile, 1915, Ann. Mag. Nat. Hist. (8) 15: 213.

Western Australian Distribution: Hibernia Reef (Bruce, 1992).
Thaumastocaris streptopus Kemp, 1922

Thaumastocaris streptopus Kemp, 1922, Rec. Indian Mus. 14: 244-247, figs 78-80.

Western Australian Distribution: Cartier and Hibernia Reefs (Bruce, 1992); central Kimberleys, Churchill Reef (Berggren, 1997c).

\section{Typton nanus Bruce, 1987}

Typton nanus Bruce, 1987, Beagle, Rec. N.T. Mus. Arts and Sci. 4 (1): 49-56, figs 1-5.

Western Australian Distribution: $16^{\circ} 34^{\prime} \mathrm{S}$. $121^{\circ} 27^{\prime}$ E (Bruce, 1987c).

\section{Typtonychus dimorphus (Bruce, 1986)}

Typton dimorphus Bruce, 1986, Crustaceana 50 (3): 278-286, figs $1-4$.

Typtonychus dimorphus. - Bruce, 1994, Theses Zoolog. 25:146.

Western Australian Distribution: Ashmore Reef (Bruce, 1986b).

Vir philippinensis Bruce and Svoboda, 1984

Vir philippinensis Bruce and Svoboda, 1984, Asian Mar. Biol. 1: 87-94, figs 1-4.

Western Australian Distribution: Hibernia Reef (Bruce, 1992).

\section{ACKNOWLEDGEMENTS}

I am most grateful to Diana Jones and Melissa Titelius for the opportunity to report on these Western Australian palaemonoid shrimps, and to Miranda Lowe, for the opportunity to examine the $P$. batei holotype. I am also most grateful to Matz Berggren for making his unpublished reports available. This study was also supported by the Australian Biological Resources Study.

\section{REFERENCES}

Audouin, V. (1826). Éxplication sommaire des planches de Crustacés de l'Égypte et de la Syrie, publiées par Jules-Cesar Savigny, membre de l'Institute: offrant un exposé des caractères naturelles des genres avec la distinction des espèces. Description de l'Égypte ou receuil des observations et des recherches qui on été faites en Égypte pendant l'expédition de l'armée francaise. Histoire Naturelle 1 (4): 77-98.

Austin, A.D. and Sale, P.F. (1980). Community Structure of the Fauna Associated with the Coral Pocillopora damicornis (L.) on the Great Barrier Reef. Australian Journal of Marine and Freshwater Research 31: 163174.

Balss, H. (1921). Stomatopoda, Macrura, Paguridea und Galatheidea. Results of Dr. E. Mjöberg's Swedish Scientific Expeditions to Australia 1910-13. XXIX Kungliga Svenska Vetenskakademiens Handlingar 61: 1-24. 
Bate, C.S. (1888). Report on the Crustacea Macrura dredged by H.M.S. Challenger during the years $1873-$ 76. Report on the Scientific Results of the Voyage of H.M.S. Challenger during the years 1873-76, Zoology, 24: i-xc, 1-942, figs. 1-76, pls. 1-150.

Berggren, M. (1997a). The shrimp fauna off the southern coast of Western Australia. 5 pp. In: Colman, J.G. (ed.), South Coast Terrestrial and Marine Reserve Integration Study, Project No: N713, Data Report: MRIP/SC/F-11/1997 Department of Conservation and Land Management, Perth. (umpublished report)

Berggren, M. (1997b). The shrimp fauna in Shark Bay, Western Australia. 6 pp. Department of Conservation and Land Management, Perth. (unpublished report)

Berggren, M. (1997c). The shrimps from the reefs off the Kimberley, Western Australia. pp. 86-90. In Walker, D.I. (ed.), Marine Biological Survey of the Central Kimberley Coast, Western Australia. University of Western Australia, (unpublished report).

Berggren, M. (1999). Notopontonia platycheles, a littleknown shallow water shrimp from Western Australia (Decapoda: Pontoniinae). Journal of Crustacean Biology 19: 180-187.

Black, R. and J. Prince. (1983). Fauna associated with the coral Pocillopora damicornis at the southern limit of its distribution in Western Australia. Journal of Biogeography, 10: 135-152.

Boone, L. (1935). Crustacea and Echinodermata, Scientific Results of the World Cruise of the Yacht "Alva", 1931, William K. Vanderbilt, Commanding. Bulletin of the Vanderbilt Marine Museum 6: 1-263.

Borradaile, L.A. (1898). A revision of the Pontoniidae. Annals and Magazine of Natural History (7) 2: 376391.

Borradaile, L.A. (1899). On some Crustaceans from the South Pacific. Part III. Macrura. Proceedings of the Zoological Society of London 1898: 1000-1015.

Borradaile, L.A. (1915). Notes on Carides. Annals and Magazine of Natural History (8) 15: 205-213.

Borradaile, L.A. (1917). On the Pontoniinae. The Percy Sladen Trust Expedition to the Indian Ocean in 1905, under the leadership of Mr J. Stanley Gardiner. Transactions of the Linnean Society of London, Zoology (2) 17: 323-396.

Bourdon, R. and Bruce, A.J. (1983). On Probynia, a new genus of bopyrid (Isopoda Epicaridea) parasitic on pontoniine shrimps from the Great Barrier Reef. Crustaceana 44: 310-316

Bray, D.M. (1976). A review of two Western Australian shrimps of the genus Palaemonetes, P. australis Dakin, 1915 and $P$. atrinubes sp. nov. Records of the Western Australian Museum 4: 65-84.

Bruce, A.J. (1968). A report on some pontoniid shrimps from New Caledonia (Crustacea Decapoda Natantia). Bulletin du Musum national d'Histoire Naturelle (2) 39(6): 1148-1171.

Bruce, A.J. (1969). Preliminary descriptions of sixteen new species of the genus Periclimenes Costa, 1844 (Crustacea, Decapoda Natantia, Pontoninae). Zoologisches Mededelingen Leiden 43: 253-278.

Bruce, A.J. (1970). Observations on the Indo-West Pacific species of the genus Palaemonella Dana, 1852 (Decapoda, Pontoniinae). Crustaceana 19: 273-287.
Bruce, A.J. (1971). Records of some rare pontoniinid shrimps from Australian waters, with remarks upon the mouthparts of some species of the genus Periclimenes Costa, 1844. Zoologische Verhandelingen, Leiden 114: 1-32.

Bruce, A.J. (1973). Gnathophylloides robustus sp. nov, a new commensal gnathophyllid shrimp from Western Australia, with the designation of a new genus Levicaris (Decapoda, Caridea) Crustaceana 24: 17-32.

Bruce, A.J. (1974). Coralliocaris viridis sp. nov., a preliminary note (Decapoda Natantia, Pontoniinae). Crustaceana,. 26: 222-224.

Bruce, A.J. (1974a). A report on a small collection of pontoniinid shrimps from the Island of Farquhar. Crustaceana 27: 189-203.

Bruce, A.J. (1974b). Observations upon some specimens of the genus Periclimenaeus Borradaile (Decapoda Natantia, Pontoniinae) originally described by G. Nobili. Bulletin du Muséum National d'Histoire Naturelle, Paris (3), no. 258, Zool. 180: 1557-1583.

Bruce, A.J. (1975). Further observations on the IndoWestPacific species of the genus Palaemonella Dana, 1852 (Decapoda Natantia, Pontoniinae). Crustaceana 29: $169-185$.

Bruce, A.J. (1976). A report on a small Collection of Pontoniine Shrimps from the Northern Indian Ocean. Journal of the Marine Biological Association of India 16 (1974): 437-545.

Bruce, A.J. (1977a). Pontoniine shrimps in the collections of the Australian Museum. Records of the Australian Museum 31: 39-81.

Bruce, A.J. (1977b). A Re-description of Periclimenes aesopius (Bate), (Crustacea, Decapoda, Pontoniinae), with Remarks on Related Species. Australian Zoology 19: 201-216.

Bruce, A.J. (1977c). A report on a small collection of pontoniinid shrimps from Queensland, Australia. Crustaceana 33: 167-181.

Bruce, A.J. (1978a). A report on a collection of pontoniine shrimps from Madagascar and adjacent seas. Zoological Journal of the Linnean Society 62: 205-290.

Bruce, A.J. (1978b). The re-examination of some pontoniine shrimp types first described by L.A. Borradaile. Crustaceana 34: 251-268.

Bruce, A.J. (1978c). Periclimenes soror Nobili, a pontoniin shrimp new to the American fauna, with observations on its Indo-WestPacific distribution. Tethys 8 (4)(1976): 299-306.

Bruce, A.J. (1979). Notes on some Indo-Pacific Pontoniinae, XXXI. Periclimenes magnificus sp. nov., a coelenterate associate from the Capricorn Islands (Decapoda, Palaemonidae). Crustaceana, Supp. 5: 195-208.

Bruce, A.J. (1980a). On some pontoniine shrimps from Nouméa, New Caledonia. Cahiers de I'Indo-Pacifique 2: $1-39$.

Bruce, A.J. (1980b). Shrimp: the complex life relationships of shrimps on the Great Barrier Reef. Geo 2: 38-53.

Bruce, A.J. (1981). Pontoniine shrimps of Heron Island Atoll Research Bulletin 245: 1-33.

Bruce, A.J. (1982a). The Pontoniine Shrimp Fauna of Hong Kong. In Morton, B. and Tseng, C.K. (eds.), Proceedings of the First International Marine 
Biological Workshop: The Marine Fauna of Hong Kong and Southern China, Hong Kong, 1980 1: 233284.

Bruce, A.J. (1982b). The Shrimps associated with IndoWest Pacific Echinoderms, with the Description of a New Species in the Genus Periclimenes Costa, 1844. (Crustacea: Pontoniinae). Australian Museum Memoir 16: 191-216.

Bruce, A.J. (1983a). The pontoniine shrimp fauna of Australia. Australian Museum Memoir 18: 195-218. (1982).

Bruce, A.J. (1983b). Additions to the marine fauna of the Northern Territory. 1. Decapod Crustacea: Caridea and Stenopodidea. The Beagle, Records of the Museums and Art Galleries of the Northern Territory 1: $41-49$.

Bruce, A.J. (1983c). Expedition Rumphius II (1975). Crustacés parasites, commensaux, etc. (Th. Monod éd.) IX. Crustacés Décapodes (I: Natantia Pontoniinae) Bulletin du Muséum National d'Histoire Naturelle, Paris (4) 5 A3: 871-902.

Bruce, A.J. (1986a). Logerende pa koralrevet. - rejer i samliv med mange andere dyr. Naturens Verden 1986: $161-167$.

Bruce, A.J. (1986b). Notes on some Indo-Pacific Pontoniinae, XLIII. A new species of Typton from Ashmore Reef, Timor Sea (Decapoda, Palaemonidae). Crustaceana 50: 278-286.

Bruce, A.J. (1987a). Three new species of commensal shrimps from Port Essington, Arnhem Land, Northern Australia (Crustacea: Decapoda: Palaemonidae). The Beagle, Records of the Museums and Art Galleries of the Northern Territory (1986) 3: 143-166, figs. 1-15.

Bruce, A.J. (1987b). Notes on some Indo-Pacific Pontoniinae, XLIV. Periclimenes darwiniensis sp. nov. from the Northern Territory, Australia (Decapoda, Caridea). Crustaceana 52: 29-39.

Bruce, A.J. (1987c). Typton nanus sp. nov., a new commensal shrimp (Crustacea: Decapoda: Palaemonidae) from the Australian North-West Shelf.). The Beagle, Records of the Museums and Art Galleries of the Northern Territory 4: 49-56.

Bruce, A.J. (1987d). Metaphryxus intutus Bruce (Crustacea: Isopoda) a bopyrid parasite new to the Australian fauna. The Beagle, Records of the Museums and Art Galleries of the Northern Territory 3: 213.

Bruce, A.J. (1988a). The shrimp fauna of a small tropical reef, the East Point Fish Reserve, Darwin. In Larson, H.K., Michie, M.G. and Hanley, J.R. (eds), Darwin Harbour, Proceedings of the Workshop on Research and Management held in Darwin, 2-3 September, 1987. A.N.U. North Australia Research Unit, Mangrove Monograph, 4: 226-245.

Bruce, A.J. (1988b). Two new palaemonid shrimps (Crustacea: Decapoda) from the Australian Northwest Shelf. Journal of Natural History 22: 1263-1276.

Bruce, A.J. (1988c). Exopontonia malleatrix, new genus, new species, a palaemonid shrimp from Ashmore Reef, Timor Sea. Journal of Crustacean Biology 8: 122 130

Bruce, A.J. (1989a). A new palaemonid shrimp from the
Zostera-beds of Moreton Bay, Queensland. The Beagle, Records of the Museums and Art Galleries of the Northern Territory 5 (1988): 105-114, figs. 1-5.

Bruce, A.J. (1989b). Notes on some Indo-Pacific Pontoniinae, XLV. Conchodytes maculatus sp. nov., a new bivalve associate from the Australian Northwest Shelf. Crustaceana 56: 182-192.

Bruce, A.J. (1990a). Recent additions to the pontoniine shrimp fauna of Australia. The Beagle, Records of the Museums and Art Galleries of the Northern Territory 7: 9-20.

Bruce, A.J. (1990b). Additions to the marine shrimp fauna of Hong Kong. In Morton, B. (ed.), Proceedings of the Second International Marine Biology Workshop: The Marine Flora and Fauna of Hong Kong and Southern China, Hong Kong, 1986 2: 611-648.

Bruce, A.J. (1990c). A new cnidarian-associated palaemonid shrimp from Port Essington, Cobourg Peninsula, Australia. Indo-Malayan Zoology 6 (1989): 229-243.

Bruce, A.J. (1991). Crustacea Decapoda: Further deep-sea Palaemonoid shrimps from New Caledonian waters. In: Crosnier, A. (ed.), Résultats des Campagnes MUSORSTOM, 9. Mémoirs du Muséum National d'Histoire Naturelle, (A) 152: 299-411.

Bruce, A.J. (1992). Crustacea: Decapoda Caridea. pp. 128131. In Russell, B.C. and Hanley, J.R. (eds), The Biological Resources and Heritage Values of the Cartier and Hibernia Reef Systems, Timor Sea. Northern Territory Museum, Darwin.

Bruce, A.J. (1998). Pontoniine shrimps from Moreton Bay, Queensland (Crustacea: Decapoda: Pontoniine). Memoirs of the Queensland Museum 42: 387-389.

Bruce, A.J. (2002). A new species of Palaemonella (Crustacea: Decapoda: Pontoniinae) from East Africa. The Beagle, Records of the Museums and Art Galleries of the Northern Territory 18: 15-18.

Bruce, A.J. (2002a). Notes on some Indo-Pacific Pontoniinae, XLVI. Palaemonella foresti sp. nov., a new pontoniine shrimp from Western Australia (Decapoda, Palaeminidae), with a review of the IndoWest Pacific species of the genus Paalaemonella Dana, 1852. Crustaceana 75: 277-298.

Bruce, A.J. (2004). A partial revision of the genus Periclimenes Costa, 1884 (Crustacea: Decapoda: Palaemonidae). Zootaxa 582: 1-27.

Bruce, A.J. (2005). New species of Periclimenaeus Borradaile (Crustacea: Decapoda: Pontoniinae) from Ashmore Reef, North Western Australia, with remarks on $P$. pachydentatus Bruce, 1969. Records of the Western Australian Museum 22: 325-342.

Bruce, A.J. and Coombes, K.E. (1995). The palaemonoid shrimp fauna (Crustacea: Decapoda: Caridea) of the Cobourg Peninsula, Northern Territory. The Beagle, Records of the Museums and Art Galleries of the Northern Territory 12: 101-144.

Bruce, A.J. and Coombes, K.E. (1997). An annotated check-list of the caridean shrimps (Crustacea: Decapoda) of Darwin Harbour, with descriptions of three new species of Periclimenes (Palaemonidae: Pontoniinae). pp 301-337. In Hanley, J.R., Caswell, G., Megirian, D. and Larson, H.K. (eds), Proceedings of the Sixth International Marine Biological 
Workshop, The Marine Flora and Fauna of Darwin Harbour, Northern Territory, Australia. Museums and Art Galleries of the Northern Territory and the Australian Marine Sciences Association, Darwin, Australia, 1997

Calman, W.T. (1939). Crustacea: Caridea. The John Murray Expedition 1933-34, ScientificReports 6: $183-$ 224.

Coleman, N. (1988). Discover Heron Island, pp. 1-64. Sea Australia Resource Centre, Brisbane.

Dana, J.D. (1852). Crustacea. United States Exploring Expedition during the years 1838, 1839, 1840, 1841, 1842 under the command of Charles Wilkes, U.S.N. 13: $1-685$.

Dana, J.D. (1855). Crustacea. United States Exploring Expedition during the years 1838, 1839, 1840, 1841, 1842 under the Command of Charles Wilkes, U.S.N. 13: atlas, $1-27$, pls. 1-96.

Davie, P.J.F. (ed.). (1998). The Wild Guide to Moreton Bay: Wildlife and Habitats of a Beautiful Australian Coast. - Noosa to the Tweed. Queensland Museum, Brisbane.

Davie, P.J.F. (2002). Crustacea: Malacostraca: Phyllocarida, Hoplocarida, Eucarida (Part 1). In Wells, A. and Houston, W.W.K. (eds) Zoological Catalogue of Australia, 19.3A. Melboume: CSIRO Publishing, Australia: xii $551 \mathrm{pp}$.

Davie, P.J.F. and Short, J.W. (1995). Part 9. Crustaceans, pp 118-126. In Wells, F.E., Hanley, J.R. and Walker, D.I. (eds), Survey of the Marine Biota of the Southern Kimberley Islands, Western Australia. Western Australian Museum, Perth.

Davie, P.J.F. and Short, J.W. (1996). Part 8. Crustaceans, pp 68-74. In Walker, D.I., Wells, F.E. and Hanley, J.R. (eds), Survey of the Marine Biota of the Eastern Kimberley, Western Australia. Western Australian Museum, Perth.

De Grave, S. (1998). Pontoniinae (Crustacea: Decapoda: Palaemonidae) associated with bivalve molluscs from Hansa Bay, Papua New Guinea. Belgian Journal of Zoology 128: 13-22.

Duris, Z., and Bruce A.J. (1995). A revision of the 'petithouarsii' species group of the genus Periclimenes Costa, 1844 (Crustacea: Decapoda: Palaemonidae). Journal of Natural History 29: 610671.

Ellis, R. (1987). Australia's Southern Seas. National Geographic 171 (3): 286-319.

Fransen, C.H.J.M. (1994). Marine palaemonid shrimps of the Netherlands Seychelles Expedition 1992-1993. Zoologische Verhandelingen, Leiden 297: 85-152.

Fujino, T, and Miyake, S. (1967). Two species of pontoniid prawns commensal with bivalves (Crustacea, Decapoda, Palaemonidae). Publications of the Seto Marine Biological Laboratory 15 (4): 291-296.

Fujino, T. and Miyake, S. (1968). Descriptions of two new species of pontonid shrimps (Crustacea, Decapoda, Palaemonidae) commensal with sponges. Ohmu, Occasional Papers of the Zoological Laboratory, Faculty of Agriculture, Kyushu University 1(3): 85-96.

Gordon, 1. (1935). On new or imperfectly known species of Crustacea Macrura, Journal of the Linnean Society of London, Zoology 39: 307-351.
Heller, C. (1861). Synopsis der in rothen Meere vorkommenden Crustaceen. Verhandlungen des Kaiserlich-königlichen Zoologisch-Botanischen Gesellschatt in Wien 11: 3-32.

Heller, C. (1862). Beitrage zur Crustaceen-Fauna des rothen Meeres. Zweiter Theil. Sitzungberichte der Mathematische-Naturwissenschaftlichen Classe der Kaiserlichen Akademie der Wissenschatten 44 (1): 241-295, pls. 1-3.

Hipeau-Jacquotte, R. (1971). Notes de faunistique et de biologie marines de Madagascar. V. Platypontonia hyotis nov. sp. (Decapoda Natantia, Pontonimae) Crustaceana 20: 125-140.

Hoedt, F.E., Choat, J.H., Collins, J. and Cruz, J.J. (2000) Mourilyan Harbour and Abbot Point Survevs: Port Marine Baseline Surveys and Surveys for Introduced Marine Pests. pp i-vi, $1-49$.

Holthuis, L.B. (1952). The Decapoda of the Siboga Expedition. Part XI. The Palaemonidae collected by the Siboga and Snellius Expeditions with remarks on other species. II. Subfamily Pontoninae. Siboga Expedition Monograph 39a 10: 1-252.

Holthuis, L.B. (1958). Contributions to the Knowledge of the Red Sea, 8. Crustacea Decapoda from the northern Red Sea (Gulf of Aqaba and Sinai Peninsula). 1 Macrura. Bulletin of the Sea Fisheries Research Station (Haifa, Israel) 17(8-9): 1-40.

Holthuis, L.B. (1959). Results of the re-examination of the type specimens of some species belonging to the subfamilies Pontoniinae and Palaemoninae (Crustacea Decapoda Macrura). Zoologisches Mededelingen, Leiden 36: 193-200.

Holthuis, L.B. (1981). Description of three new species of shrimps (Crustacea: Decapoda: Caridea) from Pacific Islands. Proceedings of the Biological Society of Washington 94: 787-800.

Jones, D.S. (1990). Annotated checklist of marine decapod Crustacea from Shark Bay, Western Australia. pp 169-208. In Berry, P.B., Bradshaw, S.D. and Wilson, B.R. (eds), Report of the France-Australe Bicentenary Expedition Committee. Western Australian Museum, Perth,

Jones, D.S., and Morgan, G.J. (1993). An annotated checklist of Crustacea from Rottnest Island, Western Australia. pp 136-162. In Wells, F.E., Walker, D.I., Kirkman, H. and Lethbridge, R. (eds), Proceedings of the Fitth International Marine Biological Workshop: The Marine Flora and Fauna of Rottnest Island, Western Australia. Western Australian Museum, Perth.

Jones, D.S. and G.J. Morgan. (2002). A Field Guide to Crustaceans of Australian Waters. Western Australian Museum, Perth and New Holland Publishers. pp 224.

Kemp, S. (1922). Notes on Crustacea Decapoda in the Indian Museum. XV. Pontoniinae. Records of the Indian Museum 24: 113-288.

Kirkman, H., Humphries, P. and Manning, R. (1991). The epibenthic tauna of seagrass beds and bare sand in Princess Roval Harbour and King George Sound, Albany, Western Australia. pp 553-563. In Wells, F.E., Walker, D.I., Kirkman, H. and Lethbridge, R. (eds), Proceedings of the Third International Marine Biological Workshop: The Marine Flora and Fauna of 
Albany Western Australia. Western Australian Museum, Perth.

Kubo, I. (1940). Studies on Japanese Palaemonoid Shrimps. II. Pontoniinae. Journal of the Imperial Fisheries Institute, Tokyo 34: 31-75.

Li, X. (1996). The pontoniine shrimps (Crustacea: Caridea: Palaemonidae) from Nansha Islands, China 1. Studies on Marine Fauna and Biogeography of the Nansha Islands and Neighbouring Waters. II. Ocean Press, Beijing. pp. 309.

Li, X. (2000). Catalog of the Genera and Species of Pontoniinae Kingsley, 1878. Xueyuan Press, Beijing. pp 319.

Man, J.G. de. (1902). Die von Herrn Professor Kükenthal im Indischen Archipel gesammelten Dekapoden und Stomatopoden. In Kükenthal, W. Ergebnisse einer zoologischen Forschungsreise in den Molukken und Borneo. Abhandlungen der Senckenbergischen Naturforschenden Gesellschaft 25: 467-929.

Marin, N.I., Britayev, T.A. and Anker, A. (2005). Pontoniine shrimps associated with cnidarians: new records and list of species from coastal waters of Viet Nam. Arthropoda Selecta 13: 199-218.

McMichael, D.F. (1963). The Swain Reefs Expedition. Australian Natural History: 210-214.

McNeill, F.A. 1926. Crustacea. The Biology of NorthWest Islet, Capricorn Group. Australian Zoology 4: 299-318.

McNeill, F.A. (1968). Crustacea, Decapoda and Stomatopoda. Great Barrier Reef Expedition, 1928-29, Scientific Reports 7: 1-98.

Miers, E.J. (1884). Crustacea. Report of the Zoological Collections made in the Indo-Pacific Ocean during the Voyage of H.M.S. "Alert" 1881-2. Part I. The collections from Melanesia. Part II. Collections from the western Indian Ocean. London: British Museum. pp. 178-322, 513-575. pls 18-32, 46-51.

Miyake, S. and Fujino, T. (1967). On four species of Pontoniinae (Crustacea, Decapoda, Palaemonidae) found in Porifera inhabiting the coastal regions of Kyushu, Japan. Journal of the Faculty of Agriculture, Kyushu University 14: 275-291.

Nobili, G. (1904). Diagnoses préliminaires de vingt-huit espèces nouvelles de Stomatopodes et Décapodes Macroures de la Mer Rouge. Bulletin du Muséum National d'Histoire Naturelle, Paris 10: 228-238.

Nobili, G. (1905). Décapodes nouveaux des côtes d'Arabie et du Golfe Persique. (Diagnoses préliminaires). Bulletin du Muséum National d'Histoire Naturelle, Paris 11: 158-164

Nobili, G. (1906). Crustacés Décapodes et Stomatopodes. Mission J. Bonnier et Ch. Perez (Golfe Persique, 1901). Bulletin scientifique de la France et de la Belgique 40 13-159.

Okuno, J. (2004). Periclimenes speciosus, a new spcies of anthozoan associated shrimp (Crustacea: Decapoda: Palaemonidae) from southern Japan. Zoological Science 21: 865-875.

Patton, W.K. (1966). Decapod crustacea commensal with Queensland branching corals. Crustaceana 10: 271295.

Patton, W.K. (1974). Community structure among the animals inhabiting the coral Pocillopora damicornis at Heron Island, Australia. In Vernberg, W.G. (ed.), Symbiosis in the Sea. Belle W. Baruch Library in Marine Science 2: 219-243.

Paul'son, O.M. (1875). Studies on the Crustacea of the Red Sea with Notes on Crustacea of the adjacent Seas. Part I. Podophthalmata and Edriophthalmata (Cumacea). The Israel Program fro Scientific Translations. pp. i-xiv, 1-144, pls 1-21.

Peters, W. (1852). Conchodytes, eine neue in Muscheln lebende Gattung von Garneelen. Bericht über die zur Bekanntmachung geeigneten Verhandlungen der K. Preuss. Akademie der Wissenschaften zu Berlin 1852: 588-595.

Potts, F.A. (1915). The fauna associated with the crinoids of a tropical coral reef: with especial reference to its colour variations. Papers from the Department of Marine Biology of the Carnegie Institute of Washington 8: 74-96.

Saville-Kent, W. (1893). The Great Barrier Reef of Australia; its products and Potentialities. pp. i-xvii, 1-387, text figs, pls 1-48, chromo pls 1-16, 1 map.

Shiino, S. M. (1942). Bopyrids from the South Sea Islands with description of a hyperparasitic cryptoniscid. Palao Tropical Biological Station Studies 2: $437-458$.

Stephenson, T.A., Stephenson, A., Tandy, G. and Spender, M. (1931). The Structure of Ecology of Low Isles and other Reefs. Scientific Reports of the Great Barrier Reef Expedition 3: 17-112.

Stimpson, W. (1860). Prodromus descriptionis animalium evertebratorum quae in Expeditione ad Oceanum Pacificum Septemtrionalem a Republica Federato missa, C. Ringgold et J. Rodgers Ducibus, Observavit et descripsit. Proceedings of the Academy of Natural Sciences Philadelphia 1860: 22-48.

Sullivan, R. (1997). Seasoned campaigner in underwater work. Geo 19: 28-38.

Tsareva, L.A. (1980). On specieses (sic) composition and ecology of decapods of the Scott Reefs, 113-130. In Preobrazhensky, B.V. and Krasnov, S.V. (eds), Biology of Coral Reefs. Moscow, pp 258.

Vytopil, E., and Willis, B.L. (2001). Epifaunal community structure in Acropora spp (Scleractinia) on the Great Barrier Reef: implications of coral morphology and habitat complexity. Coral Reefs 20: 281-288.

Wadley, V.A. (1978). A checklist and illustrated key to the epibenthic shrimps (Decapoda: Natantia) of Moreton Bay, Queensland. CSIRO Division of Fisheries and Oceanography 99: 1-24, figs 1-10.

Yokoya, Y. (1936). Some Rare and New species of Decapod Crustaceans found in the vicinity of the Misaki Marine Biological Station. Japanese Journal Zoology 7: 129-146.

Young, P.C. and Wadley, V.A. (1979). Distribution of shallow-water epibenthic macrofauna in Moreton Bay, Queensland, Australia. Marine Biology 53: 8397.

Zann, L.P. (1980). Living together in the Sea. T.F.H. Publications Inc. Ltd., Neptune, N.J, pp 1-416.

Zehntner, L. (1894). Crustacés de l'Archipel Malais. Voyage de MM. M. Bedot et C. Pictet dans I'Archipel Malais. Revue Suisse de Zoologie et Annales du Musée d'Histoire Naturelle de Genève 2: 135-214. 


\section{ADDENDUM}

Following the recent publicatins of Marin and Chan (2006), and Okuno and Fujita (2007), the following new name combinations should be noted: Periclimenes alegrias Bruce 1986 to Unguicaris alegrias (Bruce, 1986); Periclimenes amboinensis (De Man 1888) to Laomenes amboinensis (De Man, 1888), and Parapontonia nudirostris Bruce 1968 to Laomenes nudirostris (Bruce, 1968).

Marin, I.N. and Chan, T.-Y (2006). Two new genera and a new species of crinoid associated pontoniine shrimps (Crustacea: Decapoda: Palaemonidae). Journal of Crustacean Biology, 26: 524-539.

Okuno, J. and Fujita, Y. (2007). Resurrection of the genus Laomenes A. H. Clark, 1919 (Decapoda, Caridea, Palaemonidae). Crustaceana 80: 113-124. 